Homology, Homotopy and Applications, vol.14(1), 2012, pp.133-158

\title{
CUBICAL APPROACH TO DERIVED FUNCTORS
}

\author{
IRAKLI PATCHKORIA
}

\author{
(communicated by Michael A. Mandell)
}

\begin{abstract}
We construct a cubical analog of the Tierney-Vogel theory of simplicial derived functors and prove that these cubical derived functors are naturally isomorphic to their simplicial counterparts. We also show that this result generalizes the well-known fact that the simplicial and cubical singular homologies of a topological space are naturally isomorphic.
\end{abstract}

\section{Introduction}

In [24] Tierney and Vogel for any functor $F: \mathscr{C} \rightarrow \mathscr{B}$, where $\mathscr{C}$ is a category with finite limits and a projective class $\mathscr{P}$, and $\mathscr{B}$ is an abelian category, constructed simplicial derived functors and investigated relationships of their theory with other theories of derived functors. Namely, they showed that if $\mathscr{C}$ is abelian and $F$ is additive, then their theory coincides with the classical relative theory of EilenbergMoore [7], whereas if $\mathscr{C}$ is abelian and $F$ is an arbitrary functor, then it gives a generalization of the theory of Dold-Puppe $[\mathbf{6}]$. Besides, they proved that their derived functors are naturally isomorphic to the cotriple derived functors of Barr-Beck [2, 3] if there is a cotriple in $\mathscr{C}$ that realizes the given projective class $\mathscr{P}$.

Almost all homology theories from classical homological algebra like, for example, Hochschild or André-Quillen (co)homologies as well as singular homology theory of topological spaces, can be naturally viewed as special cases of Tierney-Vogel simplicial derived functors $([\mathbf{2 4},(3.1)]$ and $[\mathbf{3},(1.3),(1.4),(10.2)])$.

It is well known that classical singular homology of topological spaces (usually defined using singular simplices) can be also obtained by means of singular cubes (see e.g., $[\mathbf{1 7}],[\mathbf{2 1}]$, where the cubical approach is used). A question arises whether other homologies that are special cases of Tierney-Vogel theory admit a cubical description. The aim of this paper is to give a positive answer to this question. More precisely, using projective pseudocubical resolutions (Definition 6.10), we construct a cubical analog of the derived functor theory of Tierney-Vogel and prove the following

Theorem 1.1. Suppose $\mathscr{C}$ is a category with finite limits, $\mathscr{P}$ a projective class in $\mathscr{C}$ in the sense of [24, §2], $\mathscr{B}$ an abelian category, and $F: \mathscr{C} \rightarrow \mathscr{B}$ a functor. Let $\mathbf{L}_{n}^{\Delta} F: \mathscr{C} \rightarrow \mathscr{B}, n \geqslant 0$, be the Tierney-Vogel simplicial derived functors of $F$, and

Received September 9, 2011, revised December 5, 2011; published on June 4, 2012.

2000 Mathematics Subject Classification: 18G10, 18E25, 18G30, 18G25, 55N10.

Key words and phrases: derived functor, normalized chain complex, presimplicial object, projective class, projective pseudocubical resolution, pseudocubical object.

Article available at http://intlpress.com/HHA/v14/n1/a7 and doi:10.4310/HHA.2012.v14.n1.a7

Copyright (C) 2012, International Press. Permission to copy for private use granted. 
$\mathbf{L}_{n}^{\square} F: \mathscr{C} \rightarrow \mathscr{B}, n \geqslant 0$, the cubical derived functors of $F$ (Section 6). Then there is an isomorphism

$$
\mathbf{L}_{n}^{\Delta} F(C) \cong \mathbf{L}_{n}^{\square} F(C), \quad C \in \mathscr{C}, \quad n \geqslant 0,
$$

which is natural in $F$ and in $C$.

Before saying more on the content of the paper itself, let us say a few words about cubical objects and techniques. Simplicial methods have been developed for a long time, and are well-known and successfully used in mathematics. Cubical techniques have seen less success, but developed initially on the one hand by the systematic use of singular cubes in the singular homology theory of topological spaces (see, for example, $[\mathbf{1 7}],[\mathbf{2 1}])$, and, on the other hand, by the papers of Kan $[\mathbf{1 3}, \mathbf{1 4}]$ which have related cubical sets to homotopy theory. There are several reasons why people mostly prefer simplicial objects to the cubical ones. For example, a cubical group, in fact even a cubical abelian group can fail to satisfy the Kan condition $[\mathbf{2 2}, \mathbf{2 3}]$. (This defect is partially eliminated in $[\mathbf{2 5}]$ where it is shown that cubical groups which possess connections of [4] are Kan.) Another example is the classical bar construction which is naturally simplicial and not cubical. However, cubical objects have also a number of advantages compared to simplicial ones. A prominent example for this is a cubical homotopy which is given by a single morphism in each dimension, whereas a simplicial homotopy requires "many" morphisms.

Although cubical objects are not so heavily used in mathematics as the simplicial ones, the research (see, for example, $[\mathbf{1}, \mathbf{4}, \mathbf{5}, \mathbf{1 0}, \mathbf{1 1}, \mathbf{1 2}, \mathbf{1 6}]$ ) shows that they are nevertheless interesting and important. In the present paper we show that the cubical theory is good enough to capture almost all (co)homology theories from classical homological algebra.

Now let us list contents of the paper by sections.

In Section 2 we remind the reader of some definitions and constructions needed in the sequel. In particular, we recall the definition of a (pre)cubical object and the construction of the singular cubical set of a topological space.

Section 3 is devoted to a review of the relative Eilenberg-Moore derived functor theory of additive functors from [7].

In Section 4 we recall the theory of Tierney-Vogel and prove that the simplicial derived functors of a functor $F: \mathscr{C} \rightarrow \mathscr{B}$ (where $\mathscr{C}$ is a category with finite limits and a fixed projective class, and $\mathscr{B}$ an abelian category) are just the Eilenberg-Moore derived functors of the unique additive extension $F_{a d}: \mathbb{Z} \mathscr{C} \rightarrow \mathscr{B}$ of the functor $F$, where $\mathbb{Z} \mathscr{C}$ is the free preadditive category generated by $\mathscr{C}$. (Note that this statement is essentially due to Barr and Beck $[\mathbf{3}, \S 5]$ (see Remark 4.6).)

Next, in Section 5 we define precubical resolutions and prove some of their properties. In particular, we prove Proposition 5.3 which says that our precubical resolutions possess pseudodegeneracies. This is crucial for constructing cubical derived functors as opposed to the simplicial case (see Remark 5.4).

Section 6 is devoted to the (pseudo)cubical normalization functor. This functor is used to define the cubical derived functors at the end of the section.

The main result of the paper (Theorem 1.1) is proved in Section 7. We show that for any functor $F: \mathscr{C} \rightarrow \mathscr{B}$, where $\mathscr{C}$ is a category with finite limits and a projective class, and $\mathscr{B}$ an abelian category, the cubical derived functors of $F$ are naturally isomorphic 
to the Eilenberg-Moore derived functors of the additive extension $F_{a d}: \mathbb{Z} \mathscr{C} \rightarrow \mathscr{B}$. This together with the aforementioned statement from Section 4 (Proposition 4.5) implies Theorem 1.1.

Finally, the purpose of Section 8 is to show that Theorem 1.1 generalizes the wellknown fact that the simplicial and cubical singular homologies of a topological space are naturally isomorphic. The latter is classically proved via the method of acyclic models. We essentially also use an acyclic models argument. However, our approach provides a new conceptual explanation of the coincidence of the simplicial and cubical singular homologies of a topological space.

\section{Preliminaries}

Let us recall some definitions and constructions needed in the sequel.

Definition 2.1. A preadditive category is a category $\mathscr{A}$ together with the following data:

(i) For any objects $X, Y$ in $\mathscr{A}$, the set of morphisms $\operatorname{Hom}_{\mathscr{A}}(X, Y)$ is an abelian group.

(ii) For any morphisms $f, g: X \longrightarrow Y, h: W \longrightarrow X$ and $u: Y \longrightarrow Z$ in $\mathscr{A}$, the following hold

$$
(f+g) h=f h+g h, \quad u(f+g)=u f+u g .
$$

In other words, a preadditive category is just a ring with several objects in the sense of $[\mathbf{1 8}]$.

Definition 2.2. Let $\mathscr{A}$ be a preadditive category. A (non-negatively graded) chain complex $C$ in $\mathscr{A}$ is a sequence of objects and morphisms

$$
\cdots \longrightarrow C_{n} \stackrel{d_{n}}{\longrightarrow} C_{n-1} \longrightarrow \cdots \longrightarrow C_{2} \stackrel{d_{2}}{\longrightarrow} C_{1} \stackrel{d_{1}}{\longrightarrow} C_{0}
$$

in $\mathscr{A}$ such that $d_{n} d_{n+1}=0, n \geqslant 1$.

Definition 2.3. Let $\mathscr{A}$ be a preadditive category. An augmented chain complex over an object $A \in \mathscr{A}$ (or just a complex over $A$ ) is a sequence

$$
\cdots \longrightarrow C_{n} \stackrel{d_{n}}{\longrightarrow} C_{n-1} \longrightarrow \cdots \longrightarrow C_{2} \stackrel{d_{2}}{\longrightarrow} C_{1} \stackrel{d_{1}}{\longrightarrow} C_{0} \stackrel{d_{0}}{\longrightarrow} A
$$

in $\mathscr{A}$ such that $d_{n} d_{n+1}=0, n \geqslant 0$. In other words, an augmented chain complex in $\mathscr{A}$ is a chain complex $C$ in $\mathscr{A}$ together with a morphism $C_{0} \stackrel{d_{0}}{\longrightarrow} A$ satisfying $d_{0} d_{1}=0$.

Let $C$ and $C^{\prime}$ be chain complexes in $\mathscr{A}$. A morphism of chain complexes $f: C \rightarrow C^{\prime}$ is a sequence of morphisms $\left\{f_{n}: C_{n} \rightarrow C_{n}^{\prime}\right\}_{n \geqslant 0}$ in $\mathscr{A}$ such that $d_{n} f_{n}=f_{n-1} d_{n}^{\prime}, n \geqslant 1$. A morphism of augmented chain complexes is defined similarly.

Definition 2.4. Let $f, g: C \rightarrow C^{\prime}$ be morphisms of chain complexes in a preadditive category $\mathscr{A}$. One says that $f$ and $g$ are chain homotopic if there is a sequence $\left\{h_{n}: C_{n} \rightarrow C_{n+1}^{\prime}\right\}_{n \geqslant 0}$ of morphisms in $\mathscr{A}$ such that

$$
\begin{gathered}
d_{1}^{\prime} h_{0}=f_{0}-g_{0}, \\
d_{n+1}^{\prime} h_{n}+h_{n-1} d_{n}=f_{n}-g_{n}, \quad n \geqslant 1 .
\end{gathered}
$$


Next we recall the well-known definition:

Definition 2.5. A presimplicial object $S$ in a category $\mathscr{C}$ is a family of objects $\left(S_{n} \in\right.$ $\mathscr{C})_{n \geqslant 0}$ together with face morphisms

$$
\partial_{i}: S_{n} \rightarrow S_{n-1} \quad(n \geqslant 1,0 \leqslant i \leqslant n)
$$

in $\mathscr{C}$ satisfying

$$
\partial_{i} \partial_{j}=\partial_{j-1} \partial_{i} \quad i<j
$$

A simplicial object is a presimplicial object $S$ together with degeneracy morphisms

$$
s_{i}: S_{n} \rightarrow S_{n+1} \quad(0 \leqslant i \leqslant n)
$$

in $\mathscr{C}$ satisfying

$$
\partial_{i} s_{j}= \begin{cases}s_{j-1} \partial_{i} & i<j, \\ \mathrm{id} & i=j, \quad j+1, \\ s_{j} \partial_{i-1} & i>j+1,\end{cases}
$$

and

$$
s_{i} s_{j}=s_{j+1} s_{i} \quad i \leqslant j
$$

A morphism $f: S \rightarrow S^{\prime}$ between presimplicial objects in a category $\mathscr{C}$ is a family of morphisms $\left(f_{n}: S_{n} \rightarrow S_{n}^{\prime}\right)_{n \geqslant 0}$ in $\mathscr{C}$ which commute with the face operators. If $S$ and $S^{\prime}$ are simplicial objects, then the morphisms $f_{n}$ must commute with the face and degeneracy operators.

Let $\mathscr{C}$ be a category and $C \in \mathscr{C}$ an object. An augmented (pre) simplicial object over $C$ is a (pre)simplicial object $S$ in $\mathscr{C}$ together with a morphism $\partial: S_{0} \rightarrow C$ satisfying $\partial \partial_{0}=\partial \partial_{1}$.

Any presimplicial object in a preadditive category gives rise to a chain complex in the following way:

Definition 2.6. Let $S$ be a presimplicial object in a preadditive category $\mathscr{A}$. The unnormalized chain complex $U(S)$ associated to $S$ is defined by

$$
\begin{gathered}
U(S)_{n}=S_{n}, \quad n \geqslant 0, \\
d_{n}=\sum_{i=0}^{n}(-1)^{i} \partial_{i}: U(S)_{n} \longrightarrow U(S)_{n-1}, \quad n>0 .
\end{gathered}
$$

The presimplicial identities imply that $d^{2}=0$.

Further we recall some basic definitions from the cubical theory.

Definition 2.7. A precubical object $X$ in a category $\mathscr{C}$ is a family of objects $\left(X_{n} \in\right.$ 
$\mathscr{C})_{n \geqslant 0}$ together with morphisms

$$
\partial_{i}^{0}, \partial_{i}^{1}: X_{n} \rightarrow X_{n-1} \quad(n \geqslant 1, \quad 1 \leqslant i \leqslant n)
$$

in $\mathscr{C}$ satisfying

$$
\partial_{i}^{\alpha} \partial_{j}^{\varepsilon}=\partial_{j-1}^{\varepsilon} \partial_{i}^{\alpha} \quad i<j, \quad \alpha, \varepsilon \in\{0,1\} .
$$

The morphisms $\partial_{i}^{0}$ and $\partial_{i}^{1}$ are called face operators.

Definition $2.8([\mathbf{1 3}])$. A cubical object $X$ in a category $\mathscr{C}$ is a family of objects $\left(X_{n} \in \mathscr{C}\right)_{n \geqslant 0}$ together with morphisms

$$
\partial_{i}^{0}, \partial_{i}^{1}: X_{n} \rightarrow X_{n-1} \quad(n \geqslant 1, \quad 1 \leqslant i \leqslant n)
$$

and

$$
s_{i}: X_{n-1} \rightarrow X_{n} \quad(n \geqslant 1, \quad 1 \leqslant i \leqslant n)
$$

in $\mathscr{C}$ which satisfy

$$
\begin{gathered}
\partial_{i}^{\alpha} \partial_{j}^{\varepsilon}=\partial_{j-1}^{\varepsilon} \partial_{i}^{\alpha} \quad i<j, \quad \alpha, \varepsilon \in\{0,1\} . \\
s_{i} s_{j}=s_{j+1} s_{i} \quad i \leqslant j
\end{gathered}
$$

and

$$
\partial_{i}^{\alpha} s_{j}= \begin{cases}s_{j-1} \partial_{i}^{\alpha} & i<j, \\ \mathrm{id} & i=j, \\ s_{j} \partial_{i-1}^{\alpha} & i>j,\end{cases}
$$

where $\alpha \in\{0,1\}$. The morphisms $s_{i}$ are called degeneracy operators.

Let $\mathscr{C}$ be a category and $C$ an object in $\mathscr{C}$. An augmented (pre)cubical object over $C$ is a (pre)cubical object $X$ in $\mathscr{C}$ together with a morphism $\partial: X_{0} \rightarrow C$ satisfying $\partial \partial_{1}^{0}=\partial \partial_{1}^{1}$. We will denote such an object by $X \stackrel{\partial}{\longrightarrow} C$.

A morphism $f: X \rightarrow X^{\prime}$ between precubical objects in a category $\mathscr{C}$ is a family of morphisms $\left(f_{n}: X_{n} \rightarrow X_{n}^{\prime}\right)_{n \geqslant 0}$ in $\mathscr{C}$ which commute with the face operators. If $X$ and $X^{\prime}$ are cubical objects, then the morphisms $f_{n}$ must commute with the face and degeneracy operators. A morphism between augmented (pre)cubical objects $X \stackrel{\partial}{\longrightarrow} C$ and $X^{\prime} \stackrel{\partial^{\prime}}{\longrightarrow} C^{\prime}$ in $\mathscr{C}$ is a morphism

$$
\bar{f}=\left(f_{n}: X_{n} \rightarrow X_{n}^{\prime}\right)_{n \geqslant 0}
$$

between the (pre)cubical objects $X$ and $X^{\prime}$ together with a morphism $f: C \rightarrow C^{\prime}$ satisfying $f \partial=\partial^{\prime} f_{0}$.

Definition 2.9. Let $X$ be a precubical object in a preadditive category $\mathscr{A}$. The unnormalized chain complex $C(X)$ associated to $X$ is defined by

$$
\begin{gathered}
C(X)_{n}=X_{n}, \quad n \geqslant 0 \\
d_{n}=\sum_{i=1}^{n}(-1)^{i}\left(\partial_{i}^{1}-\partial_{i}^{0}\right): C(X)_{n} \longrightarrow C(X)_{n-1}, \quad n>0 .
\end{gathered}
$$


The precubical identities show that $d^{2}=0$.

Example 2.10. Let $I^{n}$ denote the standard $n$-cube, $n \geqslant 0$. There are standard maps

$$
\delta_{\varepsilon}^{i}: I^{n-1} \rightarrow I^{n}, \quad n \geqslant 1, \quad \varepsilon \in\{0,1\} \quad 1 \leqslant i \leqslant n,
$$

and

$$
\eta^{i}: I^{n} \rightarrow I^{n-1}, \quad n \geqslant 1, \quad 1 \leqslant i \leqslant n,
$$

defined by

$$
\delta_{\varepsilon}^{i}\left(t_{1}, \cdots, t_{n-1}\right)=\left(t_{1}, \cdots, t_{i-1}, \varepsilon, t_{i}, \cdots, t_{n-1}\right),
$$

and

$$
\eta^{i}\left(t_{1}, \cdots t_{n}\right)=\left(t_{1}, \cdots, t_{i-1}, t_{i+1}, \cdots, t_{n}\right),
$$

respectively. The spaces $\left\{I^{n}\right\}_{n \geqslant 0}$ together with these maps form the standard cocubical object in the category Top of topological spaces. This cocubical object gives rise to a classical example of a cubical object, the singular cubical set $Q(Y)$ of a topological space $Y$. The precise construction is:

$$
\begin{gathered}
Q_{n}(Y)=\operatorname{Hom}_{\mathbf{T o p}}\left(I^{n}, Y\right), \\
\partial_{i}^{\varepsilon}\left(f: I^{n} \rightarrow Y\right)=f \delta_{\varepsilon}^{i}, \quad \varepsilon \in\{0,1\}, \quad n \geqslant 1 \quad 1 \leqslant i \leqslant n, \\
s_{i}\left(f: I^{n-1} \rightarrow Y\right)=f \eta^{i}, \quad n \geqslant 1 \quad 1 \leqslant i \leqslant n .
\end{gathered}
$$

Note that $Q(Y)$ is used to define the singular cubical homology of the space $Y$ (see $[\mathbf{1 7}$, II.§2] or Example 6.9 below).

\section{Partially defined Eilenberg-Moore derived functors}

The following definitions are well-known.

Definition 3.1 ([7, I.3]). Let $\mathscr{A}$ be a preadditive category and $\mathscr{P}$ a class of objects in $\mathscr{A}$ (which need not be a "projective class" in any sense). A complex

$$
\cdots \longrightarrow C_{2} \stackrel{\partial_{2}}{\longrightarrow} C_{1} \stackrel{\partial_{1}}{\longrightarrow} C_{0} \stackrel{\partial_{0}}{\longrightarrow} A
$$

over $A \in \mathscr{A}$ is said to be $\mathscr{P}$-acyclic if for any $Q \in \mathscr{P}$ the sequence of abelian groups

$$
\cdots \longrightarrow \operatorname{Hom}_{\mathscr{A}}\left(Q, C_{1}\right) \stackrel{\partial_{1 *}}{\longrightarrow} \operatorname{Hom}_{\mathscr{A}}\left(Q, C_{0}\right) \stackrel{\partial_{0 *}}{\longrightarrow} \operatorname{Hom}_{\mathscr{A}}(Q, A) \longrightarrow 0
$$

is exact.

Definition $3.2([7$, I.3]). Let $\mathscr{A}$ be a preadditive category and $\mathscr{P}$ a class of objects in $\mathscr{A}$. A $\mathscr{P}$-resolution of an object $A \in \mathscr{A}$ is a $\mathscr{P}$-acyclic complex

$$
\cdots \longrightarrow P_{2} \stackrel{\partial_{2}}{\longrightarrow} P_{1} \stackrel{\partial_{1}}{\longrightarrow} P_{0} \stackrel{\partial_{0}}{\longrightarrow} A
$$

over $A$ with $P_{n} \in \mathscr{P}, n \geqslant 0$.

Note that an object $A \in \mathscr{A}$ need not necessarily possess a $\mathscr{P}$-resolution.

There is a comparison theorem for $\mathscr{P}$-resolutions which can be proved using the standard homological algebra arguments (see e.g., [26, 2.2.7]). More precisely, the following is valid. 
Proposition 3.3 (Comparison theorem). Let $P_{*} \longrightarrow A$ be a complex over $A \in \mathscr{A}$ consisting of objects of $\mathscr{P}$, and let $Q_{*} \longrightarrow B$ be a $\mathscr{P}$-acyclic complex. Then any morphism $f: A \longrightarrow B$ can be extended to a morphism of augmented chain complexes

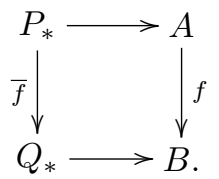

Moreover, any two such extensions are chain homotopic (see Definition 2.4).

Suppose $\mathscr{A}$ is a preadditive category, $\mathscr{P}$ a class of objects in $\mathscr{A}, \mathscr{B}$ an abelian category, $F: \mathscr{A} \longrightarrow \mathscr{B}$ an additive functor, and $\mathscr{A}^{\prime}$ the full subcategory of those objects in $\mathscr{A}$ which possess $\mathscr{P}$-resolutions. Recall that Proposition 3.3 allows one to construct the left derived functors $\mathbf{L}_{n}^{\mathscr{P}} F: \mathscr{A}^{\prime} \longrightarrow \mathscr{B}, n \geqslant 0$, of $F$ with respect to the class $\mathscr{P}$ as follows. If $A \in \mathscr{A}^{\prime}$, choose (once and for all) a $\mathscr{P}$-resolution $P_{*} \longrightarrow A$ and define

$$
\mathbf{L}_{n}^{\mathscr{P}} F(A)=H_{n}\left(F\left(P_{*}\right)\right), \quad n \geqslant 0 .
$$

Remark 3.4. If $\mathscr{P}$ is a projective class in the sense of [7], then $\mathbf{L}_{n}^{\mathscr{P}} F, n \geqslant 0$, are exactly the derived functors introduced in [7, I.3]. Note that in this case $\mathscr{A}^{\prime}=\mathscr{A}$, i.e., the functors $\mathbf{L}_{n}^{\mathscr{P}} F$ are defined everywhere.

Next we recall

Definition $3.5([7$, I.2]). Let $\mathscr{A}$ be a preadditive category and $\mathscr{P}$ a class of objects of $\mathscr{A}$. A sequence

$$
X \stackrel{f}{\longrightarrow} Y \stackrel{g}{\longrightarrow} Z
$$

in $\mathscr{A}$ is said to be $\mathscr{P}$-exact if $g f=0$ and the sequence of abelian groups

$$
\operatorname{Hom}_{\mathscr{A}}(P, X) \stackrel{f_{*}}{\longrightarrow} \operatorname{Hom}_{\mathscr{A}}(P, Y) \stackrel{g_{*}}{\longrightarrow} \operatorname{Hom}_{\mathscr{A}}(P, Z)
$$

is exact for any $P \in \mathscr{P}$.

Definition 3.6 ([7, I.2]). A closure of a class $\mathscr{P}$, denoted by $\overline{\mathscr{P}}$, is the class of all those objects $Q \in \mathscr{A}$ for which

$$
\operatorname{Hom}_{\mathscr{A}}(Q, X) \stackrel{f_{*}}{\longrightarrow} \operatorname{Hom}_{\mathscr{A}}(Q, Y) \stackrel{g_{*}}{\longrightarrow} \operatorname{Hom}_{\mathscr{A}}(Q, Z)
$$

is exact whenever $X \stackrel{f}{\longrightarrow} Y \stackrel{g}{\longrightarrow} Z$ is $\mathscr{P}$-exact.

Clearly, $\mathscr{P} \subseteq \overline{\mathscr{P}}$ and $\mathscr{P}$-exactness is equivalent to $\overline{\mathscr{P}}$-exactness. In particular, $\overline{\overline{\mathscr{P}}}=\overline{\mathscr{P}}$.

Note that if a preadditive category $\mathscr{A}$ has a terminal object, then any $\mathscr{P}$-resolution

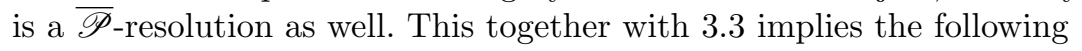

Proposition 3.7. Let $\mathscr{A}$ be a preadditive category with a terminal object, $\mathscr{P}$ a class of objects in $\mathscr{A}, \mathscr{B}$ an abelian category, $F: \mathscr{A} \longrightarrow \mathscr{B}$ an additive functor, and $A$ an object in $\mathscr{A}$ which possesses a $\mathscr{P}$-resolution. Then there is a natural isomorphism

$$
\mathbf{L}_{n}^{\mathscr{P}} F(A) \cong \mathbf{L}_{n}^{\overline{\mathscr{P}}} F(A), \quad n \geqslant 0 .
$$


IRAKLI PATCHKORIA

\section{Simplicial derived functors and Eilenberg-Moore derived functors}

In this section we review the construction of simplicial derived functors from $[\mathbf{2 4}$, $\S 2]$ and show that they can be obtained as derived functors of an additive functor.

Definition 4.1. Let $\mathscr{P}$ be a class of objects in a category $\mathscr{C}$.

(i) A morphism $f: C \rightarrow C^{\prime}$ in $\mathscr{C}$ is said to be $\mathscr{P}$-epimorphic if

$$
\operatorname{Hom}_{\mathscr{C}}(P, f): \operatorname{Hom}_{\mathscr{C}}(P, C) \rightarrow \operatorname{Hom}_{\mathscr{C}}\left(P, C^{\prime}\right)
$$

is surjective for all $P \in \mathscr{P}$.

(ii) The class $\mathscr{P}$ is called a projective class if for each $C \in \mathscr{C}$ there exists a $\mathscr{P}$ epimorphism $e: P \rightarrow C$ with $P \in \mathscr{P}$.

Definition $4.2([24,(2.1)])$. Let $\mathscr{C}$ be a category and

$$
f_{0}, f_{1}, \cdots, f_{n}: C \rightarrow D
$$

a sequence of morphisms in $\mathscr{C}, n \geqslant 0$. A simplicial kernel of the sequence $\left(f_{0}, f_{1}, \cdots\right.$, $\left.f_{n}\right)$ is a sequence

$$
k_{0}, k_{1}, \cdots, k_{n+1}: K \rightarrow C
$$

such that

(i) $f_{i} k_{j}=f_{j-1} k_{i}$ for $0 \leqslant i<j \leqslant n+1$,

(ii) if $h_{0}, h_{1}, \cdots, h_{n+1}: L \rightarrow C$ is any other sequence satisfying identities $f_{i} h_{j}=$ $f_{j-1} h_{i}$ for $0 \leqslant i<j \leqslant n+1$, then there exists a unique morphism $h: L \rightarrow K$ in $\mathscr{C}$ with $k_{i} h=h_{i}, 0 \leqslant i \leqslant n+1$.

Let $\mathscr{P}$ be a projective class in $\mathscr{C}, S \stackrel{\partial}{\longrightarrow} C$ an augmented presimplicial object over $C \in \mathscr{C}$, and suppose that $\mathscr{C}$ has finite limits. There is a factorization $([\mathbf{2 4},(2.3)])$

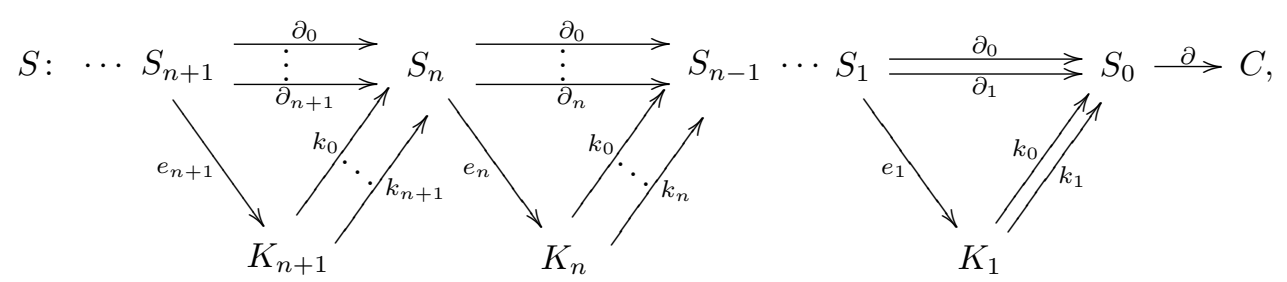

where $K_{1} \underset{k_{1}}{\stackrel{k_{0}}{\longrightarrow}} S_{0}$ is a kernel pair of $S_{0} \stackrel{\partial}{\longrightarrow} C$, and

$$
k_{0}, k_{1}, \cdots, k_{n}: K_{n} \rightarrow S_{n-1}
$$

a simplicial kernel of $\left(\partial_{0}, \cdots, \partial_{n-1}\right)$ for $n \geqslant 2$.

One says that $S \stackrel{\partial}{\longrightarrow} C$ is a $\mathscr{P}$-projective presimplicial resolution if $S_{n} \in \mathscr{P}$, $n \geqslant 0$, and $\partial$ and $e_{n}, n \geqslant 1$, are $\mathscr{P}$-epimorphic.

Note that if $\mathscr{C}$ is a category with finite limits and a projective class $\mathscr{P}$, then any $C \in \mathscr{C}$ has a $\mathscr{P}$-projective resolution. 
Now we are ready to recall the construction of simplicial derived functors. Let $\mathscr{C}$ be a category with finite limits, $\mathscr{P}$ a projective class, $\mathscr{B}$ an abelian category, and $F: \mathscr{C} \longrightarrow \mathscr{B}$ a functor. The simplicial derived functors $\mathbf{L}_{n}^{\Delta} F$ of $F$ with respect to the class $\mathscr{P}$ are defined as follows $[\mathbf{2 4}, \S 2]$. For any object $C \in \mathscr{C}$, choose (once and for all) a $\mathscr{P}$-projective presimplicial resolution

$$
S \stackrel{\partial}{\longrightarrow} C
$$

of $C$ and define

$$
\mathbf{L}_{n}^{\Delta} F(C)=H_{n}(U(F(S))), \quad n \geqslant 0 .
$$

By the comparison theorem for projective presimplicial resolutions $[\mathbf{2 4},(2.4)$ Theorem], the objects $\mathbf{L}_{n}^{\Delta} F(C)$ are well-defined and functorial in $F$ and $C$.

We will now show that the derived functors $\mathbf{L}_{n}^{\Delta} F$ can be obtained as the derived functors of some additive functor. First recall

Lemma 4.3. Let $S \stackrel{\partial_{0}}{\longrightarrow} S_{-1}$ be an augmented presimplicial set. Suppose that the map $\partial_{0}: S_{0} \longrightarrow S_{-1}$ is surjective and the following extension condition holds: For any $n \geqslant 0$ and any collection of $n+2$ elements $x_{i} \in S_{n}, 0 \leqslant i \leqslant n+1$, satisfying

$$
\partial_{i} x_{j}=\partial_{j-1} x_{i}, \quad 0 \leqslant i<j \leqslant n+1,
$$

there exists $x \in S_{n+1}$ such that $\partial_{i} x=x_{i}, 0 \leqslant i \leqslant n+1$. Then the augmented chain complex

$$
U(\mathbb{Z}[S]) \longrightarrow \mathbb{Z}\left[S_{-1}\right]
$$

is chain contractible $(\mathbb{Z}[X]$ denotes the free abelian group generated by $X)$. In particular, it has trivial homology in each dimension.

The proof is standard (one constructs inductively a presimplicial contraction).

Example 4.4. Let $S \stackrel{\partial}{\longrightarrow} C$ be a $\mathscr{P}$-projective presimplicial resolution of $C$ and suppose $P \in \mathscr{P}$. Then the augmented presimplicial set

$$
\operatorname{Hom}_{\mathscr{C}}(P, S) \stackrel{\partial_{*}}{\longrightarrow} \operatorname{Hom}_{\mathscr{C}}(P, C)
$$

satisfies the conditions of 4.3. Consequently, the homologies of the augmented chain complex

$$
U\left(\mathbb{Z}\left[\operatorname{Hom}_{\mathscr{C}}(P, S)\right]\right) \longrightarrow \mathbb{Z}\left[\operatorname{Hom}_{\mathscr{C}}(P, C)\right]
$$

vanish.

Now suppose again that $\mathscr{C}$ is a category with finite limits, $\mathscr{P}$ a projective class in $\mathscr{C}, \mathscr{B}$ an abelian category, and $F: \mathscr{C} \longrightarrow \mathscr{B}$ a functor. Let $\mathbb{Z} \mathscr{C}$ denote the free preadditive category generated by $\mathscr{C}[\mathbf{1 8}, \S 1]$, i.e., the objects of $\mathbb{Z} \mathscr{C}$ are those of $\mathscr{C}$, and for any objects $C$ and $D$ in $\mathscr{C}, \operatorname{Hom}_{\mathbb{Z} \mathscr{C}}(C, D)$ is the free abelian group generated by $\operatorname{Hom}_{\mathscr{C}}(C, D)$. The composition of morphisms in $\mathbb{Z} \mathscr{C}$ is induced by that in $\mathscr{C}$. Clearly, $\mathscr{C}$ is a subcategory of $\mathbb{Z} \mathscr{C}$. Further, since the category $\mathscr{B}$ is abelian (and 
therefore additive), the functor $F: \mathscr{C} \longrightarrow \mathscr{B}$ can be uniquely extended to an additive functor

$$
F_{a d}: \mathbb{Z} \mathscr{C} \longrightarrow \mathscr{B} .
$$

The following proposition relates the simplicial derived functors of $F$ to the EilenbergMoore derived functors of $F_{a d}$.

Proposition 4.5. Suppose $\mathscr{C}$ is a category with finite limits, $\mathscr{P}$ a projective class in $\mathscr{C}, \mathscr{B}$ an abelian category, and $F: \mathscr{C} \longrightarrow \mathscr{B}$ a functor. Then:

(i) For any $\mathscr{P}$-projective presimplicial resolution $S \longrightarrow C$, the augmented chain complex

$$
U(S) \longrightarrow C
$$

in $\mathbb{Z} \mathscr{C}$ is a $\mathscr{P}$-resolution of $C$ in the sense of Definition 3.2.

(ii) For any $C \in \mathscr{C}$, there is a natural isomorphism

$$
\mathbf{L}_{n}^{\Delta} F(C) \cong \mathbf{L}_{n}^{\mathscr{P}} F_{a d}(C), \quad n \geqslant 0 .
$$

Proof. The first claim immediately follows from 4.4 and the definition of $\mathbb{Z} \mathscr{C}$. The second claim is a consequence of the first one and the definition of $F_{a d}$. Indeed, if $S \longrightarrow C$ is a $\mathscr{P}$-projective presimplicial resolution of $C$, then we have

$$
\mathbf{L}_{n}^{\Delta} F(C)=H_{n}(U(F(S)))=H_{n}\left(F_{a d}(U(S))\right)=\mathbf{L}_{n}^{\mathscr{P}} F_{a d}(C) .
$$

Remark 4.6. Proposition 4.5 is essentially due to Barr and Beck [3, §5]. More precisely, in the case when the projective class $\mathscr{P}$ comes from a cotriple (see $[\mathbf{2 4}, \S 3]$ ) the above statement is proved in $[\mathbf{3}, \S 5]$. (The cotriple derived functor theory of BarrBeck is a special case of the Tierney-Vogel theory $[\mathbf{2 4}, \S 3]$.) Thus 4.5 is a simple generalization of the result of Barr and Beck.

\section{Cubical resolutions}

This section is devoted to the construction and properties of cubical resolutions which we use to define cubical derived functors in Section 6.

Definition 5.1. Let $\mathscr{C}$ be a category and

$$
f_{1}^{0}, \ldots, f_{n}^{0}, f_{1}^{1}, \ldots, f_{n}^{1}: C \rightarrow D
$$

a sequence of morphisms in $\mathscr{C}, n \geqslant 1$. A cubical kernel of the sequence $\left(f_{1}^{0}, \ldots, f_{n}^{0}, f_{1}^{1}\right.$, $\left.\ldots, f_{n}^{1}\right)$ is a sequence

$$
k_{1}^{0}, \ldots, k_{n}^{0}, k_{n+1}^{0}, k_{1}^{1}, \ldots, k_{n}^{1}, k_{n+1}^{1}: K \rightarrow C
$$

of morphisms such that

(i) $f_{i}^{\varepsilon} k_{j}^{\alpha}=f_{j-1}^{\alpha} k_{i}^{\varepsilon}$ for $1 \leqslant i<j \leqslant n+1, \varepsilon, \alpha \in\{0,1\}$;

(ii) if $h_{1}^{0}, \ldots, h_{n}^{0}, h_{n+1}^{0}, h_{1}^{1}, \ldots, h_{n}^{1}, h_{n+1}^{1}: L \rightarrow C$ is any other sequence satisfying identities $f_{i}^{\varepsilon} h_{j}^{\alpha}=f_{j-1}^{\alpha} h_{i}^{\varepsilon}$ for $1 \leqslant i<j \leqslant n+1, \varepsilon, \alpha \in\{0,1\}$, then there exists a unique morphism $h: L \rightarrow K$ in $\mathscr{C}$ with $k_{i}^{\varepsilon} h=h_{i}^{\varepsilon}, 1 \leqslant i \leqslant n+1, \varepsilon \in\{0,1\}$. 
It immediately follows from the definition that the cubical kernel is just a special kind of finite limit.

Now suppose $\mathscr{C}$ is a category with finite limits, let $\mathscr{P}$ be a projective class in $\mathscr{C}$ (see Definition 4.1), and $X \stackrel{\partial}{\longrightarrow} C$ be an augmented precubical object over $C \in \mathscr{C}$. Then we have a factorization

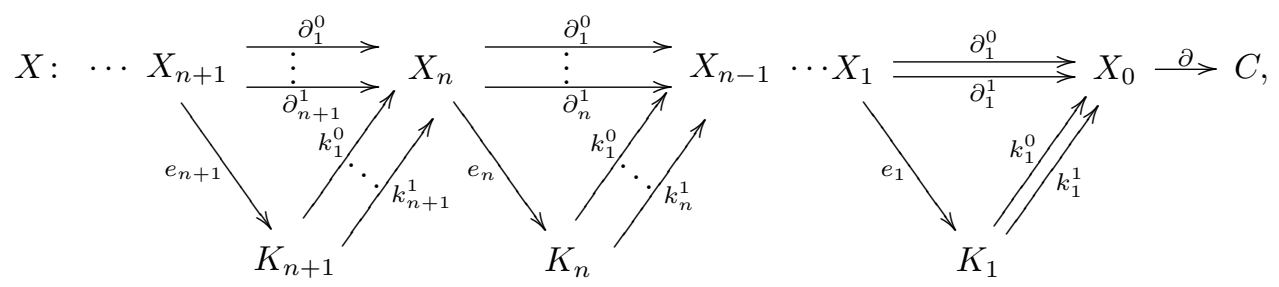

where $K_{1} \underset{k_{1}^{1}}{\stackrel{k_{1}^{0}}{\longrightarrow}} X_{0}$ is a kernel pair of $X_{0} \stackrel{\partial}{\longrightarrow} C$, and

$$
k_{1}^{0}, \ldots, k_{n}^{0}, k_{1}^{1}, \ldots, k_{n}^{1}: K_{n} \rightarrow X_{n-1}
$$

is a cubical kernel of $\left(\partial_{1}^{0}, \ldots, \partial_{n-1}^{0}, \partial_{1}^{1}, \ldots, \partial_{n-1}^{1}\right)$ for $n \geqslant 2$. We say that

1) $X \stackrel{\partial}{\longrightarrow} C$ is $\mathscr{P}$-projective if each $X_{n} \in \mathscr{P}$;

2) $\quad X \stackrel{\partial}{\longrightarrow} C$ is $\mathscr{P}$-exact if $\partial$ and $e_{n}(n \geqslant 1)$ are $\mathscr{P}$-epimorphic;

3) $\quad X \stackrel{\partial}{\longrightarrow} C$ is a $\mathscr{P}$-projective resolution of $C$ if it is $\mathscr{P}$-projective and $\mathscr{P}$-exact.

Obviously, if $\mathscr{C}$ is a category with finite limits and a projective class $\mathscr{P}$, then each $C \in \mathscr{C}$ has a $\mathscr{P}$-projective resolution. Moreover, the following comparison theorem shows that such a resolution is unique up to precubical homotopy equivalence.

Theorem 5.2. Let $X \stackrel{\partial}{\longrightarrow} C$ be $\mathscr{P}$-projective and $X^{\prime} \stackrel{\partial^{\prime}}{\longrightarrow} C^{\prime}$ be $\mathscr{P}$-exact. Then any morphism $f: C \rightarrow C^{\prime}$ in $\mathscr{C}$ can be extended to a precubical morphism

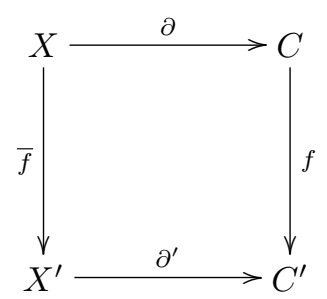

over $f$ (i.e., $\bar{f}$ and $f$ form a morphism of augmented precubical objects). Furthermore, any two such extensions are precubically homotopic. That is, if $\bar{f}, \bar{g}: X \rightarrow X^{\prime}$ are two extensions of $f$, then there exist morphisms $h_{n}: X_{n} \rightarrow X_{n+1}^{\prime}, n \geqslant 0$, in $\mathscr{C}$ such that

$$
\begin{aligned}
& \partial_{1}^{0} h_{n}=f_{n}, \quad \partial_{1}^{1} h_{n}=g_{n}, \quad n \geqslant 0, \\
& \partial_{i}^{\varepsilon} h_{n}=h_{n-1} \partial_{i-1}^{\varepsilon}, \quad n \geqslant 1, \quad 1<i \leqslant n+1, \quad \varepsilon \in\{0,1\} .
\end{aligned}
$$

Proof. We construct the extension $\bar{f}=\left(f_{n}: X_{n} \rightarrow X_{n}^{\prime}\right)$ and show its uniqueness up to precubical homotopy by induction on $n$. Since $X_{0}$ is $\mathscr{P}$-projective and $\partial^{\prime}: X_{0}^{\prime} \rightarrow$ 
$C^{\prime}$ is $\mathscr{P}$-epimorphic, there is $f_{0}: X_{0} \rightarrow X_{0}^{\prime}$ with $\partial^{\prime} f_{0}=f \partial$. Next, one has $\partial^{\prime} f_{0} \partial_{1}^{0}=$ $f \partial \partial_{1}^{0}=f \partial \partial_{1}^{1}=\partial^{\prime} f_{0} \partial_{1}^{1}$. Therefore $f_{0} \partial_{1}^{0}=k_{1}^{0} \varphi_{1}$ and $f_{0} \partial_{1}^{1}=k_{1}^{1} \varphi_{1}$ for a uniquely defined $\varphi_{1}: X_{1} \rightarrow K_{1}^{\prime}$. As $X_{1}$ is $\mathscr{P}$-projective and $e_{1}: X_{1}^{\prime} \rightarrow K_{1}^{\prime}$ is $\mathscr{P}_{\text {-epimorphic, there }}$ exists $f_{1}: X_{1} \rightarrow X_{1}^{\prime}$ with $e_{1} f_{1}=\varphi_{1}$, and we have $\partial_{1}^{0} f_{1}=k_{1}^{0} e_{1} f_{1}=k_{1}^{0} \varphi_{1}=f_{0} \partial_{1}^{0}$ and $\partial_{1}^{1} f_{1}=k_{1}^{1} e_{1} f_{1}=k_{1}^{1} \varphi_{1}=f_{0} \partial_{1}^{1}$. Thus $f_{0}$ and $f_{1}$ are constructed. Inductively, suppose given morphisms $f_{r}: X_{r} \rightarrow X_{r}^{\prime}$ for $r \leqslant n$ so that $\partial_{i}^{\omega} f_{r}=f_{r-1} \partial_{i}^{\omega}, 1 \leqslant i \leqslant r, \omega \in\{0,1\}$. Then

$$
\partial_{i}^{\omega} f_{n} \partial_{j}^{\alpha}=f_{n-1} \partial_{i}^{\omega} \partial_{j}^{\alpha}=f_{n-1} \partial_{j-1}^{\alpha} \partial_{i}^{\omega}=\partial_{j-1}^{\alpha} f_{n} \partial_{i}^{\omega}, 1 \leqslant i<j \leqslant n+1, \omega, \alpha \in\{0,1\} .
$$

Hence there is a unique $\varphi_{n+1}: X_{n+1} \rightarrow K_{n+1}^{\prime}$ such that $k_{i}^{\omega} \varphi_{n+1}=f_{n} \partial_{i}^{\omega}, 1 \leqslant i \leqslant n+$ $1, \omega \in\{0,1\}$. Since $X_{n+1}$ is $\mathscr{P}$-projective and $e_{n+1}: X_{n+1}^{\prime} \rightarrow K_{n+1}^{\prime}$ is $\mathscr{P}$-epimorphic, there exists $f_{n+1}: X_{n+1} \rightarrow X_{n+1}^{\prime}$ with $e_{n+1} f_{n+1}=\varphi_{n+1}$. Then we have $\partial_{i}^{\omega} f_{n+1}=$ $k_{i}^{\omega} e_{n+1} f_{n+1}=k_{i}^{\omega} \varphi_{n+1}=f_{n} \partial_{i}^{\omega}, 1 \leqslant i \leqslant n+1, \omega \in\{0,1\}$. This completes the inductive step and proves the existence of $\bar{f}$.

Now suppose $\bar{g}=\left(g_{n}: X_{n} \rightarrow X_{n}^{\prime}\right)$ is another extension of $f: C \rightarrow C^{\prime}$. We want to construct $h=\left(h_{n}: X_{n} \rightarrow X_{n+1}^{\prime}\right)$ with $\partial_{1}^{0} h_{n}=f_{n}, \partial_{1}^{1} h_{n}=g_{n}, n \geqslant 0$, and $\partial_{i}^{\varepsilon} h_{n}=$ $h_{n-1} \partial_{i-1}^{\varepsilon}, 1<i \leqslant n+1, \varepsilon \in\{0,1\}$. For $n=0$ consider diagram

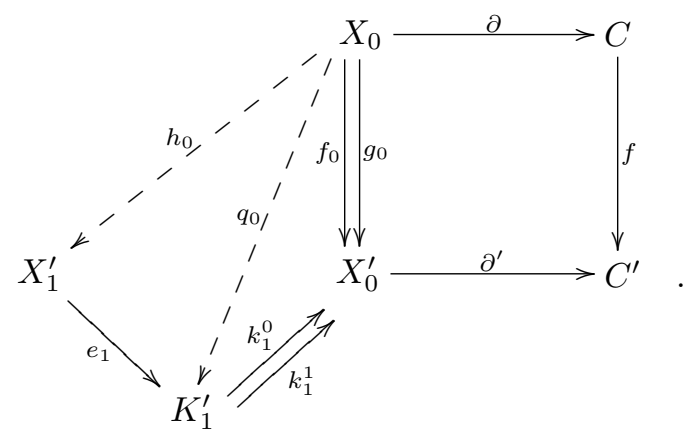

As $\partial^{\prime} f_{0}=\partial^{\prime} g_{0}$, there exists $q_{0}: X_{0} \rightarrow K_{1}^{\prime}$ such that $k_{1}^{0} q_{0}=f_{0}$ and $k_{1}^{1} q_{0}=g_{0}$. Next, since $X_{0}$ is $\mathscr{P}$-projective and $e_{1}$ is $\mathscr{P}$-epimorphic, there is $h_{0}: X_{0} \rightarrow X_{1}^{\prime}$ with $e_{1} h_{0}=$ $q_{0}$. This and the two previous equalities give $\partial_{1}^{0} h_{0}=k_{1}^{0} e_{1} h_{0}=k_{1}^{0} q_{0}=f_{0}$ and $\partial_{1}^{1} h_{0}=$ $k_{1}^{1} e_{1} h_{0}=k_{1}^{1} q_{0}=g_{0}$. Thus $h_{0}$ is constructed. Inductively, suppose given $h_{0}, h_{1}, \ldots$, $h_{n-1}$ with the required properties. (See Figure 1.) Define $\psi_{i}^{\varepsilon}: X_{n} \rightarrow X_{n}^{\prime}, 1 \leqslant i \leqslant$ $n+1, \varepsilon \in\{0,1\}$, as follows:

$$
\psi_{1}^{0}=f_{n}, \quad \psi_{1}^{1}=g_{n}, \quad \psi_{i}^{\varepsilon}=h_{n-1} \partial_{i-1}^{\varepsilon}, \quad 1<i \leqslant n+1, \quad \varepsilon \in\{0,1\} .
$$

By the induction assumption,

$$
\begin{aligned}
& \partial_{1}^{0} \psi_{j}^{\varepsilon}=\partial_{1}^{0} h_{n-1} \partial_{j-1}^{\varepsilon}=f_{n-1} \partial_{j-1}^{\varepsilon}=\partial_{j-1}^{\varepsilon} f_{n}=\partial_{j-1}^{\varepsilon} \psi_{1}^{0}, \\
& 1<j \leqslant n+1, \quad \varepsilon \in\{0,1\}, \quad n \geqslant 1, \\
& \partial_{1}^{1} \psi_{j}^{\varepsilon}=\partial_{1}^{1} h_{n-1} \partial_{j-1}^{\varepsilon}=g_{n-1} \partial_{j-1}^{\varepsilon}=\partial_{j-1}^{\varepsilon} g_{n}=\partial_{j-1}^{\varepsilon} \psi_{1}^{1}, \\
& 1<j \leqslant n+1, \quad \varepsilon \in\{0,1\}, \quad n \geqslant 1, \\
& \partial_{i}^{\alpha} \psi_{j}^{\varepsilon}=\partial_{i}^{\alpha} h_{n-1} \partial_{j-1}^{\varepsilon}=h_{n-2} \partial_{i-1}^{\alpha} \partial_{j-1}^{\varepsilon}=h_{n-2} \partial_{j-2}^{\varepsilon} \partial_{i-1}^{\alpha}=\partial_{j-1}^{\varepsilon} h_{n-1} \partial_{i-1}^{\alpha} \\
& =\partial_{j-1}^{\varepsilon} \psi_{i}^{\alpha}, \quad 1<i<j \leqslant n+1, \quad \alpha, \varepsilon \in\{0,1\}, \quad n>1 .
\end{aligned}
$$

That is $\partial_{i}^{\alpha} \psi_{j}^{\varepsilon}=\partial_{j-1}^{\varepsilon} \psi_{i}^{\alpha}$ for $1 \leqslant i<j \leqslant n+1, \alpha, \varepsilon \in\{0,1\}$. Therefore $k_{i}^{\varepsilon} q_{n}=\psi_{i}^{\varepsilon}, 1 \leqslant$ 


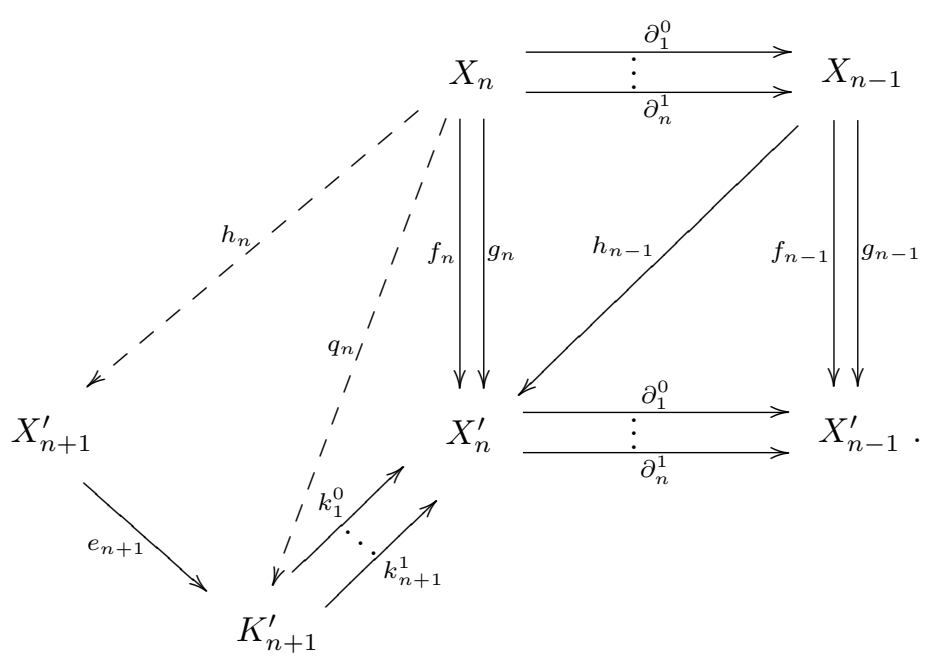

Figure 1: The inductive step in the construction of precubical homotopy.

$i \leqslant n+1, \varepsilon \in\{0,1\}$, for a uniquely defined $q_{n}: X_{n} \rightarrow K_{n+1}^{\prime}$. Since $X_{n}$ is $\mathscr{P}$-projective and $e_{n+1}$ is $\mathscr{P}$-epimorphic, there is $h_{n}: X_{n} \rightarrow X_{n+1}^{\prime}$ with $e_{n+1} h_{n}=q_{n}$. Now we have

$$
\begin{aligned}
& \partial_{1}^{0} h_{n}=k_{1}^{0} e_{n+1} h_{n}=k_{1}^{0} q_{n}=\psi_{1}^{0}=f_{n}, \\
& \partial_{1}^{1} h_{n}=k_{1}^{1} e_{n+1} h_{n}=k_{1}^{1} q_{n}=\psi_{1}^{1}=g_{n}, \\
& \partial_{i}^{\varepsilon} h_{n}=k_{i}^{\varepsilon} e_{n+1} h_{n}=k_{i}^{\varepsilon} q_{n}=\psi_{i}^{\varepsilon}=h_{n-1} \partial_{i-1}^{\varepsilon}, \\
& 1<i \leqslant n+1, \quad \varepsilon \in\{0,1\} .
\end{aligned}
$$

This finishes the inductive step and completes the proof of the theorem.

The following proposition is crucial for constructing our cubical derived functors.

Proposition 5.3. Suppose that

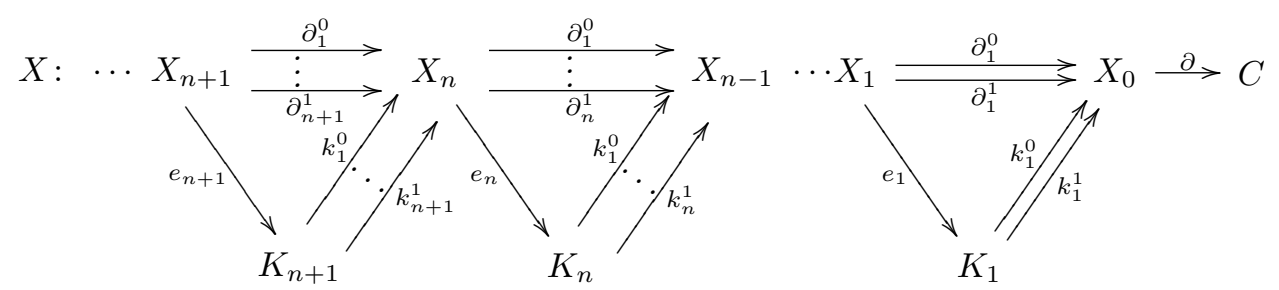

is a $\mathscr{P}$-projective resolution of $C \in \mathscr{C}$. Then $X$ has pseudodegeneracy operators, i.e., there exist $s_{i}: X_{n} \rightarrow X_{n+1}, n \geqslant 0,1 \leqslant i \leqslant n+1$, satisfying

$$
\partial_{i}^{\alpha} s_{j}= \begin{cases}s_{j-1} \partial_{i}^{\alpha} & i<j, \\ \mathrm{id} & i=j, \\ s_{j} \partial_{i-1}^{\alpha} & i>j,\end{cases}
$$

where $\alpha \in\{0,1\}$. 
Proof. As $K_{1} \underset{k_{1}^{1}}{\stackrel{k_{1}^{0}}{\longrightarrow}} X_{0}$ is a kernel pair of $X_{0} \stackrel{\partial}{\rightarrow} A$, there is $\varphi_{1}: X_{0} \rightarrow K_{1}$ such that $k_{1}^{0} \varphi_{1}=$ id and $k_{1}^{1} \varphi_{1}=$ id. On the other hand, since $X_{0}$ is $\mathscr{P}$-projective and $e_{1}$ is $\mathscr{P}$ epimorphic, $e_{1} s_{1}=\varphi_{1}$ for some $s_{1}: X_{0} \rightarrow X_{1}$ and we have $\partial_{1}^{0} s_{1}=k_{1}^{0} e_{1} s_{1}=k_{1}^{0} \varphi_{1}=$ id and $\partial_{1}^{1} s_{1}=k_{1}^{1} e_{1} s_{1}=k_{1}^{1} \varphi_{1}=$ id. Thus $s_{1}: X_{0} \rightarrow X_{1}$ is constructed. Inductively, suppose given $s_{1}: X_{0} \rightarrow X_{1}, s_{1}, s_{2}: X_{1} \rightarrow X_{2}, \ldots, s_{1}, \ldots, s_{n}: X_{n-1} \rightarrow X_{n}$ with the required properties. Fix $j, 1 \leqslant j \leqslant n+1$, and define $\psi_{i j}^{\varepsilon}: X_{n} \rightarrow X_{n}, 1 \leqslant i \leqslant n+1$, $\varepsilon \in\{0,1\}$, by

$$
\psi_{i j}^{\varepsilon}= \begin{cases}\text { id } & i=j, \\ s_{j-1} \partial_{i}^{\varepsilon} & i<j, \\ s_{j} \partial_{i-1}^{\varepsilon} & i>j .\end{cases}
$$

Using the induction assumption, one checks that $\partial_{i}^{\varepsilon} \psi_{m j}^{\alpha}=\partial_{m-1}^{\alpha} \psi_{i j}^{\varepsilon}$ for $1 \leqslant i<m \leqslant$ $n+1, \varepsilon, \alpha \in\{0,1\}$. Then there exists $\varphi_{j}: X_{n} \rightarrow K_{n+1}$ such that $k_{i}^{\varepsilon} \varphi_{j}=\psi_{i j}^{\varepsilon}, 1 \leqslant$ $i \leqslant n+1, \varepsilon \in\{0,1\}$. Since $X_{n}$ is $\mathscr{P}$-projective and $e_{n+1}$ is $\mathscr{P}$-epimorphic, there is $s_{j}: X_{n} \rightarrow X_{n+1}$ with $e_{n+1} s_{j}=\varphi_{j}$. According to this, for $i=1, \ldots, n+1$ and $\varepsilon \in$ $\{0,1\}$, one has

$$
\partial_{i}^{\varepsilon} s_{j}=k_{1}^{\varepsilon} e_{n+1} s_{j}=k_{i}^{\varepsilon} \varphi_{j}=\psi_{i j}^{\varepsilon}= \begin{cases}\text { id } & i=j, \\ s_{j-1} \partial_{i}^{\varepsilon} & i<j, \\ s_{j} \partial_{i-1}^{\varepsilon} & i>j .\end{cases}
$$

Thus we have constructed $s_{1}, \ldots, s_{n+1}: X_{n} \rightarrow X_{n+1}$ with the desired properties.

Remark 5.4. Suppose $\mathscr{C}$ is a category with finite limits, $\mathscr{P}$ a projective class in $\mathscr{C}$, $\mathscr{B}$ an abelian category, and $F: \mathscr{C} \longrightarrow \mathscr{B}$ a functor. As we will see in Section 6, Proposition 5.3 is essential for constructing the cubical derived functors of $F: \mathscr{C} \longrightarrow$ $\mathscr{B}$. This contrasts with the fact that the construction of the derived functors by Tierney and Vogel does not use the existence of pseudodegeneracies in $\mathscr{P}$-projective presimplicial resolutions. On the other hand, as shown in $[\mathbf{2 2}]$, the unnormalized chain complex functor $C$ (see Definition 2.9) sends precubically homotopic morphisms of precubical objects to chain homotopic morphisms. This together with Theorem 5.2 allows us to conclude that one does not need Proposition 5.3 to prove that the functors

$$
\bar{L}_{n}^{\square} F(C)=H_{n}(C(F(X))), n \geqslant 0,
$$

where $X$ is a $\mathscr{P}$-projective precubical resolution of $C \in \mathscr{C}$, are well defined. But in this way one obtains "wrong" derived functors by the following reason. One can easily see that $\bar{L}_{n}^{\square} F(Q) \cong F(Q)$ for any $Q \in \mathscr{P}$ and any $n \geqslant 0$. Thus in general the higher $(n>0)$ derived functors $\bar{L}_{n}^{\square} F$ do not vanish on $\mathscr{P}$-projectives, i.e., the crucial property of derived functors is not satisfied. In order to obtain reasonable derived functors one has to use a normalization procedure (just as in the case of cubical singular homology [17, II.§2] which we discuss in detail in the next section).

\section{Pseudocubical objects in idempotent complete preadditive categories and cubical derived functors}

We start with the following essential definitions. 
Definition 6.1. A pseudocubical object $\mathrm{X}$ in a category $\mathscr{C}$ is a family of objects $\left(X_{n} \in \mathscr{C}\right)_{n \geqslant 0}$ together with face operators

$$
\partial_{i}^{0}, \partial_{i}^{1}: X_{n} \longrightarrow X_{n-1}, \quad n \geqslant 1, \quad 1 \leqslant i \leqslant n,
$$

and pseudodegeneracy operators

$$
s_{i}: X_{n-1} \longrightarrow X_{n}, \quad n \geqslant 1, \quad 1 \leqslant i \leqslant n,
$$

satisfying the pseudocubical identities

$$
\partial_{i}^{\alpha} \partial_{j}^{\varepsilon}=\partial_{j-1}^{\varepsilon} \partial_{i}^{\alpha} \quad i<j, \quad \alpha, \varepsilon \in\{0,1\},
$$

and

$$
\partial_{i}^{\alpha} s_{j}= \begin{cases}s_{j-1} \partial_{i}^{\alpha} & i<j, \\ \operatorname{id} & i=j, \\ s_{j} \partial_{i-1}^{\alpha} & i>j,\end{cases}
$$

for $\alpha \in\{0,1\}$.

Note that, in general, the pseudodegeneracies need not satisfy the identities

$$
s_{i} s_{j}=s_{j+1} s_{i}, \quad i \leqslant j
$$

(see Definition 2.8).

Definition 6.2. Let $\mathscr{C}$ be a category and $C \in \mathscr{C}$ an object. An augmented pseudocubical object over $C$ is a pseudocubical object $X$ in $\mathscr{C}$ together with a morphism $\partial: X_{0} \rightarrow C$ satisfying $\partial \partial_{1}^{0}=\partial \partial_{1}^{1}$.

Important examples of augmented pseudocubical objects are provided by Proposition 5.3.

Now we recall the definition and some properties of the normalized chain complex of a pseudocubical object in the general setting of idempotent complete preadditive categories. (Note that the normalized chain complex of a cubical object in an abelian category was originally introduced by Świątek in $[\mathbf{2 2}]$.)

Definition 6.3 (see e.g., [8], [15]). A preadditive category $\mathscr{A}$ is idempotent complete if any idempotent $p: E \longrightarrow E$ in $\mathscr{A}$ (i.e., $p^{2}=p$ ) has a kernel. That is, there is a morphism

$$
i: \operatorname{Ker}(p) \longrightarrow E
$$

with $p i=0$, and for any morphism $f: F \rightarrow E$, satisfying $p f=0$, there is a unique morphism $F \stackrel{g}{\longrightarrow} \operatorname{Ker}(p)$ such that $i g=f$.

The following two propositions are well known (see e.g., [15]). 
Proposition 6.4. Suppose $\mathscr{A}$ is an idempotent complete preadditive category and $p: E \rightarrow E$ an idempotent in $\mathscr{A}$. Then there is a diagram

$$
\operatorname{Ker}(p) \underset{i^{1}}{\stackrel{\pi^{1}}{>}} E \underset{i^{2}}{\stackrel{\pi^{2}}{\rightleftarrows}} \operatorname{Ker}(1-p)
$$

such that

$$
\begin{aligned}
\pi^{1} i^{1}=1, & \pi^{2} i^{2}=1, \\
\pi^{1} i^{2}=0, & \pi^{2} i^{1}=0, \\
i^{1} \pi^{1}=1-p, & i^{2} \pi^{2}=p .
\end{aligned}
$$

In particular, the coproduct $\operatorname{Ker}(p) \oplus \operatorname{Ker}(1-p)$ exists in $\mathscr{A}$ and is isomorphic to $E$.

Proposition 6.5. Let $\mathscr{A}$ be a preadditive category. Then there exists an idempotent complete preadditive category $\mathscr{\mathscr { A }}$ (called the idempotent completion of $\mathscr{A}$ ) and a full additive embedding

$$
\varphi: \mathscr{A} \rightarrow \widetilde{\mathscr{A}}
$$

satisfying the following universal property: For any idempotent complete preadditive category $\mathscr{D}$ and an additive functor $\mathscr{A} \stackrel{\psi}{\longrightarrow} \mathscr{D}$, there is an additive functor $\psi^{\prime}: \widetilde{\mathscr{A}} \rightarrow \mathscr{D}$ which makes the diagram

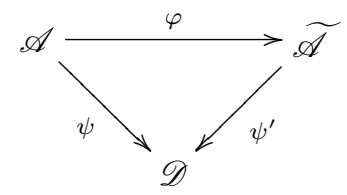

commute up to a natural equivalence, and which is unique up to a natural isomorphism.

Let $X$ be a pseudocubical object in an idempotent complete preadditive category $\mathscr{D}$. We want to construct the normalized chain complex of $X$ as a kernel of a certain idempotent endomorphism of the unnormalized chain complex $C(X)$ (see 2.9). Define

$$
\sigma_{n}^{X}=\left(1-s_{1} \partial_{1}^{1}\right)\left(1-s_{2} \partial_{2}^{1}\right) \cdots\left(1-s_{n} \partial_{n}^{1}\right): X_{n} \rightarrow X_{n}, \quad n \geqslant 0, \quad\left(\sigma_{0}=1\right) .
$$

By the pseudocubical identities $(1 \leqslant i \leqslant n)$,

$$
\begin{aligned}
\partial_{i}^{1} \sigma_{n}^{X} & =\partial_{i}^{1}\left(1-s_{1} \partial_{1}^{1}\right)\left(1-s_{2} \partial_{2}^{1}\right) \cdots\left(1-s_{n} \partial_{n}^{1}\right) \\
& =\left(1-s_{1} \partial_{1}^{1}\right) \partial_{i}^{1}\left(1-s_{2} \partial_{2}^{1}\right) \cdots\left(1-s_{n} \partial_{n}^{1}\right)=\cdots \\
& =\left(1-s_{1} \partial_{1}^{1}\right) \cdots\left(1-s_{i-1} \partial_{i-1}^{1}\right) \partial_{i}^{1}\left(1-s_{i} \partial_{i}^{1}\right) \cdots\left(1-s_{n} \partial_{n}^{1}\right) \\
& =\left(1-s_{1} \partial_{1}^{1}\right) \cdots\left(1-s_{i-1} \partial_{i-1}^{1}\right)\left(\partial_{i}^{1}-\partial_{i}^{1}\right)\left(1-s_{i+1} \partial_{i+1}^{1}\right) \cdots\left(1-s_{n} \partial_{n}^{1}\right)=0
\end{aligned}
$$

This implies

$$
\left(1-s_{i} \partial_{i}^{1}\right) \sigma_{n}^{X}=\sigma_{n}^{X}
$$

for $1 \leqslant i \leqslant n$, and thus

$$
\begin{array}{r}
\sigma_{n}^{X} \sigma_{n}^{X}=\left(1-s_{1} \partial_{1}^{1}\right)\left(1-s_{2} \partial_{2}^{1}\right) \cdots\left(1-s_{n} \partial_{n}^{1}\right) \sigma_{n}^{X}= \\
\left(1-s_{1} \partial_{1}^{1}\right)\left(1-s_{2} \partial_{2}^{1}\right) \cdots\left(1-s_{n-1} \partial_{n-1}^{1}\right) \sigma_{n}^{X}=\cdots=\left(1-s_{1} \partial_{1}^{1}\right) \sigma_{n}^{X}=\sigma_{n}^{X} .
\end{array}
$$


Further, another routine calculation using pseudocubical identities shows that the morphisms $\sigma_{n}^{X}, n \geqslant 0$, are compatible with the differentials of $C(X)$. Hence one gets an idempotent endomorphism of $C(X)$ which we denote by

$$
\sigma^{X}: C(X) \rightarrow C(X) .
$$

Since the category $\mathscr{D}$ is idempotent complete, the chain map $\sigma^{X}$ has a kernel $\operatorname{Ker} \sigma^{X}$ in the category of non-negative chain complexes in $\mathscr{D}$. Furthermore, by 6.4 , there is a diagram in the category of chain complexes

$$
\operatorname{Ker}\left(\sigma^{X}\right) \underset{i^{1}}{\stackrel{\pi^{1}}{\rightleftarrows}} C(X) \underset{i^{2}}{\stackrel{\pi^{2}}{\rightleftarrows}} \operatorname{Ker}\left(1-\sigma^{X}\right)
$$

such that

$$
\begin{array}{r}
\pi^{1} i^{1}=1, \quad \pi^{2} i^{2}=1, \\
\pi^{1} i^{2}=0, \quad \pi^{2} i^{1}=0, \\
i^{1} \pi^{1}=1-\sigma^{X}, \quad i^{2} \pi^{2}=\sigma^{X} .
\end{array}
$$

Definition 6.6. Let $X$ be a pseudocubical object in an idempotent complete preadditive category $\mathscr{D}$. The chain complex $\operatorname{Ker}\left(1-\sigma^{X}\right)$, denoted by $N(X)$, is called the normalized chain complex of $X$.

Remark 6.7. If $\mathscr{D}$ is an abelian category, then $N(X)$ admits the following description:

$$
\begin{aligned}
& N(X)_{0}=X_{0}, \quad N(X)_{n}=\bigcap_{i=1}^{n} \operatorname{Ker}\left(\partial_{i}^{1}\right), \quad n>0, \\
& d_{n}=\sum_{i=1}^{n}(-1)^{i+1} \partial_{i}^{0}: N(X)_{n} \rightarrow N(X)_{n-1}, \quad n>0 .
\end{aligned}
$$

Thus in the abelian case one does not need pseudodegeneracies to define $N(X)$.

The functor $N$ sends precubically homotopic morphisms to chain homotopic morphisms. This is proved in [22] for cubical objects in abelian categories. The proof, which we repeat here for completeness, shows that in fact one has:

Proposition 6.8. Let $X$ be a pseudocubical object and $Y$ a precubical object in an abelian category, and $f, g: X \rightarrow Y$ precubical morphisms. If $f$ and $g$ are precubically homotopic (see Proposition 5.2), then $N(f)$ and $N(g): N(X) \rightarrow N(Y)$ are chain homotopic.

Proof. Let $h=\left(h_{n}: X_{n} \rightarrow Y_{n+1}\right)_{n \geqslant 0}$ be a precubical homotopy from $f$ to $g$. For simplicity, we denote by $i_{n}: N_{n}(X) \rightarrow X_{n}$ (without the superscript 2), $n \geqslant 0$, the $n$-th level of the canonical morphism $N(X) \stackrel{i^{2}}{\longrightarrow} X$. One checks that $\partial_{i}^{1}\left(h_{n}-\right.$ $\left.g_{n+1} s_{1}\right) i_{n}=0,1 \leqslant i \leqslant n+1, n \geqslant 0$. Consequently, we have morphisms

$$
t_{n}=\left(h_{n}-g_{n+1} s_{1}\right) i_{n}: N_{n}(X) \rightarrow N_{n+1}(Y), \quad n \geqslant 0 .
$$

Clearly,

$$
d_{1} t_{0}=\partial_{1}^{0}\left(h_{0}-g_{1} s_{1}\right) i_{0}=\left(f_{0}-g_{0}\right) i_{0}=N_{0}(f)-N_{0}(g) .
$$


Furthermore, for all $n \geqslant 1$, we have

$$
\begin{aligned}
d t_{n}+t_{n-1} d= & \left(\sum_{i=1}^{n+1}(-1)^{i+1} \partial_{i}^{0}\right)\left(h_{n}-g_{n+1} s_{1}\right) i_{n} \\
& +\left(h_{n-1}-g_{n} s_{1}\right)\left(\sum_{i=1}^{n}(-1)^{i+1} \partial_{i}^{0}\right) i_{n} \\
= & f_{n} i_{n}-g_{n} i_{n}+\left(\sum_{i=2}^{n+1}(-1)^{i+1} \partial_{i}^{0}\right)\left(h_{n}-g_{n+1} s_{1}\right) i_{n} \\
& +\left(\sum_{i=1}^{n}(-1)^{i+1} \partial_{i+1}^{0}\right)\left(h_{n}-g_{n+1} s_{1}\right) i_{n} \\
= & f_{n} i_{n}-g_{n} i_{n}+\left(\sum_{i=2}^{n+1}(-1)^{i+1} \partial_{i}^{0}\right)\left(h_{n}-g_{n+1} s_{1}\right) i_{n} \\
& +\left(\sum_{i=2}^{n+1}(-1)^{i} \partial_{i}^{0}\right)\left(h_{n}-g_{n+1} s_{1}\right) i_{n} \\
= & f_{n} i_{n}-g_{n} i_{n}=N_{n}(f)-N_{n}(g) .
\end{aligned}
$$

Hence $t=\left(t_{n}: N_{n}(X) \rightarrow N_{n+1}(Y)\right)_{n \geqslant 0}$ is a chain homotopy from $N(f)$ to $N(g)$.

Example 6.9. Let $Y$ be a topological space and $\mathbb{Z}[Q(Y)]$ denote the free cubical abelian group generated by the singular cubical set $Q(Y)$ (see 2.10). Then the homology $H_{n}(N(\mathbb{Z}[Q(Y)])), n \geqslant 0$, is the $n$-th cubical singular homology of the space $Y$.

Definition 6.10. Suppose $\mathscr{C}$ is a category with finite limits, $\mathscr{P}$ a projective class in $\mathscr{C}$ and $X \stackrel{\partial}{\longrightarrow} C$ an augmented pseudocubical object in $\mathscr{C}$. One says that $X \stackrel{\partial}{\longrightarrow} C$ is a $\mathscr{P}$-projective pseudocubical resolution of $C$ if $X \stackrel{\partial}{\longrightarrow} C$ is a precubical resolution of $C$ after forgetting the pseudodegeneracies.

Let $\mathscr{C}$ be a category with finite limits, $\mathscr{P}$ a projective class in $\mathscr{C}, \mathscr{B}$ an abelian category, and $F: \mathscr{C} \longrightarrow \mathscr{B}$ a functor. Then the cubical derived functors $\mathbf{L}_{n}^{\square} F$ of $F$ with respect to the class $\mathscr{P}$ are defined as follows. For any object $C \in \mathscr{C}$, choose (once and for all) a $\mathscr{P}$-projective pseudocubical resolution

$$
X \stackrel{\partial}{\longrightarrow} C
$$

of $C$ (Existence of such a resolution follows from 5.3.) and define

$$
\mathbf{L}_{n}^{\square} F(C)=H_{n}(N(F(X))), \quad n \geqslant 0 .
$$

Theorem 5.2 and the homotopy invariance of the functor $N$ (Proposition 6.8) imply that the objects $\mathbf{L}_{n}^{\square} F(C)$ are well-defined and functorial in $F$ and $C$. In particular, we see that Proposition 5.3 is essential for constructing the cubical derived functors since pseudodegeneracies are needed in the proof of 6.8 .

The following lemma is the main technical tool for proving a cubical analog of Proposition 4.5. 
Lemma 6.11. Let $F: \mathscr{D} \rightarrow \mathscr{D}^{\prime}$ be an additive functor between idempotent complete preadditive categories. Then for any pseudocubical object $X$ in $\mathscr{D}$, there is a natural isomorphism

$$
F(N(X)) \cong N(F(X))
$$

of chain complexes in $\mathscr{D}^{\prime}$.

Proof. Applying the additive functor $F$ to the diagram

$$
\operatorname{Ker}\left(\sigma^{X}\right) \underset{i^{1}}{\stackrel{\pi^{1}}{\longrightarrow}} C(X) \underset{i^{2}}{\stackrel{\pi^{2}}{\rightleftarrows}} \operatorname{Ker}\left(1-\sigma^{X}\right)=N(X),
$$

we get a diagram

$$
F\left(\operatorname{Ker}\left(\sigma^{X}\right)\right) \underset{F\left(i^{1}\right)}{\stackrel{F\left(\pi^{1}\right)}{\longrightarrow}} C(F(X)) \underset{F\left(i^{2}\right)}{\stackrel{F\left(\pi^{2}\right)}{\gtrless}} F(N(X))
$$

(in $\mathscr{D}^{\prime}$ ) whose morphisms satisfy the following identities:

$$
\begin{array}{r}
F\left(\pi^{1}\right) F\left(i^{1}\right)=1, \quad F\left(\pi^{2}\right) F\left(i^{2}\right)=1, \\
F\left(\pi^{1}\right) F\left(i^{2}\right)=0, \quad F\left(\pi^{2}\right) F\left(i^{1}\right)=0, \\
F\left(i^{1}\right) F\left(\pi^{1}\right)=1-F\left(\sigma^{X}\right), \\
F\left(i^{2}\right) F\left(\pi^{2}\right)=F\left(\sigma^{X}\right) .
\end{array}
$$

Besides, it follows from the additivity of $F$ that $F\left(\sigma^{X}\right)=\sigma^{F(X)}$, and hence we obtain

$$
F\left(i^{2}\right) F\left(\pi^{2}\right)=\sigma^{F(X)} .
$$

This finally implies that

$$
F(N(X)) \cong \operatorname{Ker}\left(1-\sigma^{F(X)}\right)=N(F(X)) .
$$

\section{Cubical derived functors and Eilenberg-Moore derived functors}

Let $\mathscr{C}$ be a category with finite limits, $\mathscr{P}$ a projective class, $\mathscr{B}$ an abelian category, and $F: \mathscr{C} \rightarrow \mathscr{B}$ a functor. In this section we prove that for any object $C \in \mathscr{C}$, there is a natural isomorphism

$$
\mathbf{L}_{n}^{\square} F(C) \cong \mathbf{L}_{n}^{\mathscr{P}} F_{a d}(C), \quad n \geqslant 0,
$$

where $F_{\text {ad }}: \mathbb{Z} \mathscr{C} \longrightarrow \mathscr{B}$ is the additivization of $F$ (see Section 4 ). This together with 4.5 obviously implies Theorem 1.1.

The proof of this isomorphism is similar to that of 4.5. However, the arguments become a little bit complicated in the cubical setting as we have to consider normalized chain complexes in order to get the "right" homology.

Proposition 7.1. Suppose $\mathscr{A}$ is a preadditive category, $\mathscr{P}$ a class of objects in $\mathscr{A}$, $\mathscr{B}$ an abelian category, and $F: \mathscr{A} \longrightarrow \mathscr{B}$ an additive functor. Suppose further that $\overline{\mathscr{P}}$ is the closure of the class $\mathscr{P}$ in the idempotent completion $\widetilde{\mathscr{A}}$ of $\mathscr{A}$ (see 6.5), and 
$\widetilde{F}: \widetilde{\mathscr{A}} \rightarrow \mathscr{B}$ the extension of $F$. Then for any $A \in \mathscr{A}$ which possesses a $\mathscr{P}$-resolution, there is a natural isomorphism

$$
\mathbf{L}_{n}^{\mathscr{P}} F(A) \cong \mathbf{L}_{n}^{\bar{P}} \widetilde{F}(A), \quad n \geqslant 0 .
$$

Proof. Since $\widetilde{\mathscr{A}}$ has a zero object, any $\mathscr{P}$-resolution in $\mathscr{A}$ is a $\overline{\mathscr{P}}$-resolution in $\widetilde{\mathscr{A}}$. The rest follows from 3.7.

Corollary 7.2. Assume that $\mathscr{C}$ is a category with finite limits, $\mathscr{P}$ a projective class in $\mathscr{C}, \mathscr{B}$ an abelian category, and $F: \mathscr{C} \longrightarrow \mathscr{B}$ a functor. Assume further that $\widetilde{F_{a d}}: \widetilde{\mathbb{Z C}} \rightarrow \mathscr{B}$ is the extension of $F_{a d}: \mathbb{Z} \mathscr{C} \longrightarrow \mathscr{B}$ to the idempotent completion $\widetilde{\mathbb{Z C}}$ of $\mathbb{Z} \mathscr{C}$, and $\overline{\mathscr{P}}$ the closure of $\mathscr{P}$ in $\overline{\mathbb{Z} \mathscr{C}}$. Then for any object $C \in \mathscr{C}$, there is a natural isomorphism

$$
\mathbf{L}_{n}^{\mathscr{P}} F_{a d}(C) \cong \mathbf{L}_{n}^{\overline{\mathscr{P}}} \widetilde{F_{a d}}(C), \quad n \geqslant 0 .
$$

Next we need the following technical lemma:

Lemma 7.3. Let $X \stackrel{\partial}{\longrightarrow} X_{-1}$ be an augmented pseudocubical set. Suppose that $\partial: X_{0} \longrightarrow X_{-1}$ is surjective and the following conditions hold:

(i) For any $x, y \in X_{0}$, satisfying $\partial x=\partial y$, there exists $z \in X_{1}$ such that $\partial_{1}^{0} z=x$ and $\partial_{1}^{1} z=y$.

(ii) For any $n \geqslant 1$ and any collection of $2 n+2$ elements $x_{i}^{\varepsilon} \in X_{n}, 1 \leqslant i \leqslant n+1$, $\varepsilon \in\{0,1\}$, satisfying

$$
\partial_{i}^{\alpha} x_{j}^{\varepsilon}=\partial_{j-1}^{\varepsilon} x_{i}^{\alpha}, \quad 1 \leqslant i<j \leqslant n+1, \quad \alpha, \varepsilon \in\{0,1\},
$$

there exists $x \in X_{n+1}$, such that

$$
\partial_{i}^{\varepsilon} x=x_{i}^{\varepsilon}, \quad 1 \leqslant i \leqslant n+1, \quad \varepsilon \in\{0,1\} .
$$

Then the augmented normalized chain complex

$$
N(\mathbb{Z}[X]) \rightarrow \mathbb{Z}\left[X_{-1}\right]
$$

is chain contractible and therefore it has trivial homology in each dimension.

We omit the routine details of the proof here. Note only that the main idea is to construct inductively a precubical homotopy equivalence between $X$ and the constant cubical object determined by $X_{-1}$ and then use the homotopy invariance of the functor $N$ (Proposition 6.8).

Example 7.4. Let $X \stackrel{\partial}{\longrightarrow} C$ be a $\mathscr{P}$-projective pseudocubical resolution of $C$ and $P$ an object from $\mathscr{P}$. Then the augmented pseudocubical set

$$
\operatorname{Hom}_{\mathscr{C}}(P, X) \stackrel{\partial_{*}}{\longrightarrow} \operatorname{Hom}_{\mathscr{C}}(P, C)
$$

satisfies the conditions of 7.3. Consequently, the homologies of the augmented chain complex

$$
N\left(\mathbb{Z}\left[\operatorname{Hom}_{\mathscr{C}}(P, X)\right]\right) \longrightarrow \mathbb{Z}\left[\operatorname{Hom}_{\mathscr{C}}(P, C)\right]
$$

vanish. 
We are now ready to prove the main result of this section.

Proposition 7.5. Suppose $\mathscr{C}$ is a category with finite limits, $\mathscr{P}$ a projective class in $\mathscr{C}, \mathscr{B}$ an abelian category, and $F: \mathscr{C} \longrightarrow \mathscr{B}$ a functor. Then:

(i) For any $\mathscr{P}$-projective pseudocubical resolution $X \rightarrow C$, the augmented chain complex

$$
N(X) \rightarrow C
$$

in the category $\widetilde{\mathbb{Z C}}$ is a $\overline{\mathscr{P}}$-resolution of $C$ in the sense of 3.2. $\overline{\mathscr{P}}$ is the closure of $\mathscr{P}$ in $\widetilde{\mathbb{Z C}}$.)

(ii) For any $C \in \mathscr{C}$, there is a natural isomorphism

$$
\mathbf{L}_{n}^{\square} F(C) \cong \mathbf{L}_{n}^{\mathscr{P}} F_{a d}(C), \quad n \geqslant 0 .
$$

Proof. Since $N(X)_{n}$ is a retract of $X_{n}$ and $\overline{\mathscr{P}}$ is closed under retracts, $N(X)_{n} \in \overline{\mathscr{P}}$ for all $n \geqslant 0$. Further, by Lemma 6.11, one has a natural isomorphism of augmented chain complexes

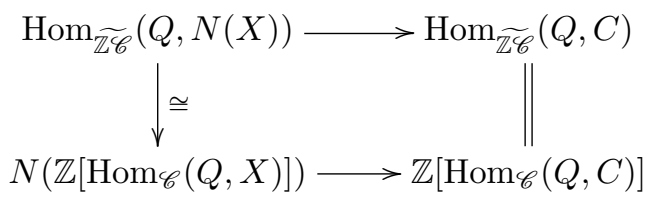

for any $Q \in \mathscr{P}$. It follows from 7.4 that the lower chain complex is acyclic and thus so is the upper one. Consequently, the augmented chain complex $N(X) \rightarrow C$ in $\widetilde{\mathbb{Z C}}$ is $\mathscr{P}$-acyclic or, equivalently, $\bar{P}$-acyclic. This completes the proof of (i).

Let us prove (ii). By Corollary 7.2, it suffices to get a natural isomorphism

$$
\mathbf{L}_{n}^{\square} F(C) \cong \mathbf{L}_{n}^{\bar{P}} \widetilde{F_{a d}}(C) .
$$

Choose any $\mathscr{P}$-projective pseudocubical resolution $X \rightarrow C$. Lemma 6.11 together with (i) gives

$$
\mathbf{L}_{n}^{\square} F(C)=H_{n}(N(F(X)))=H_{n}\left(N\left(\widetilde{F_{a d}}(X)\right)\right) \cong H_{n}\left(\widetilde{F_{a d}}(N(X))\right)=\mathbf{L}_{n}^{\overline{\mathscr{P}}} \widetilde{F_{a d}}(C) .
$$

Clearly, Theorem 1.1 is an immediate consequence of 4.5 and 7.5 .

\section{Connection with topology}

In this section we show that Theorem 1.1 generalizes the well-known fact that the cubical and simplicial singular homologies of a topological space are naturally isomorphic (see 6.9). (For basic properties of the cubical singular homology see, for example, [17].) For this we need spectral sequences of bipseudocubical objects, (pseudo)cubical analogs of spectral sequences of bi(pseudo)simplicial objects (see e.g., [9, IV.2]). Note that the identification of $E^{2}$-terms in the (pseudo)cubical setting is technically a little bit complicated compared to its simplicial counterpart as one has to take care of the normalizations.

Before starting to discuss spectral sequences we recall the following 
Definition 8.1. A bipseudocubical object $\mathrm{X}$ in a category $\mathscr{C}$ is a family of objects $\left(X_{n m} \in \mathscr{C}\right)_{n, m \geqslant 0}$ together with horizontal and vertical face operators

$$
\begin{gathered}
\partial_{i}^{0, h}, \partial_{i}^{1, h}: X_{n m} \longrightarrow X_{n-1, m}, \quad n \geqslant 1, \quad 1 \leqslant i \leqslant n, \quad m \geqslant 0, \\
\partial_{i}^{0, v}, \partial_{i}^{1, v}: X_{n m} \longrightarrow X_{n, m-1}, \quad m \geqslant 1, \quad 1 \leqslant i \leqslant n, \quad n \geqslant 0,
\end{gathered}
$$

and horizontal and vertical pseudodegeneracy operators

$$
\begin{aligned}
& s_{i}^{h}: X_{n-1, m} \longrightarrow X_{n m}, \quad n \geqslant 1, \quad 1 \leqslant i \leqslant n, \quad m \geqslant 0, \\
& s_{i}^{v}: X_{n, m-1} \longrightarrow X_{n m}, \quad m \geqslant 1, \quad 1 \leqslant i \leqslant m, \quad n \geqslant 0,
\end{aligned}
$$

such that horizontal and vertical operators commute and the pseudocubical identities (6.1) hold horizontally and vertically. If, in addition, the pseudodegeneracies are degeneracies (see 2.8) in each direction, then we say that X is a bicubical object.

Let $X$ be a bipseudocubical object in an abelian category $\mathscr{A}$. Then there is an associated first quadrant double complex $N N X$ of $X$ defined as follows:

$$
\begin{aligned}
& N N X_{00}=X_{00}, \quad N N X_{n 0}=\bigcap_{i=1}^{n} \operatorname{Ker}\left(\partial_{i}^{1, h}\right), \quad n>0, \\
& N N X_{0 m}=\bigcap_{i=1}^{m} \operatorname{Ker}\left(\partial_{i}^{1, v}\right), \quad m>0, \\
& N N X_{n m}=\bigcap_{i=1}^{n} \operatorname{Ker}\left(\partial_{i}^{1, h}\right) \cap \bigcap_{i=1}^{m} \operatorname{Ker}\left(\partial_{i}^{1, v}\right), \quad n>0, \quad m>0, \\
& d^{h}=\sum_{i=1}^{n}(-1)^{i+1} \partial_{i}^{0, h}: N N X_{n m} \rightarrow N N X_{n-1, m}, \quad n>0, \quad m \geqslant 0, \\
& d^{v}=\sum_{i=1}^{m}(-1)^{n+i+1} \partial_{i}^{0, v}: N N X_{n m} \rightarrow N N X_{n, m-1}, \quad n \geqslant 0, \quad m>0 .
\end{aligned}
$$

Proposition 8.2. Let $X$ be a bipseudocubical object in an abelian category. Then there are two first quadrant spectral sequences

$$
{ }^{I} E_{p q}^{2}=H_{p}^{h}\left(N^{h} H_{q}^{v}\left(N^{v} X\right)\right) \Longrightarrow H_{p+q}(\operatorname{Tot} N N X),
$$

and

$$
{ }^{I I} E_{p q}^{2}=H_{p}^{v}\left(N^{v} H_{q}^{h}\left(N^{h} X\right)\right) \Longrightarrow H_{p+q}(\operatorname{Tot} N N X) .
$$

(Here $N^{h}$ and $N^{v}$ stand for the horizontal and vertical normalizations, respectively, and Tot $N N X$ denotes the total complex of $N N X$.)

Proof. These spectral sequences are the two spectral sequences associated to the double complex $N N X$ (see e.g., $[\mathbf{2 6}, 5.6]$ ). By Lemma 6.11, normalization and homology in different directions commute. This gives the desired identifications of $E^{2}$-terms.

Example 8.3. Suppose $E \stackrel{f}{\longrightarrow} B$ is a Serre fibration. Let $Q_{p q}(f)$ denote the set of 
all commutative diagrams of the form

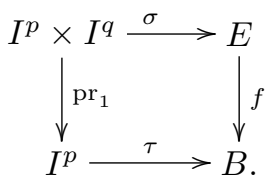

The sets $\left(Q_{p q}(f)\right)_{p, q \geqslant 0}$ together with the operators

$$
\begin{gathered}
\partial_{i}^{\varepsilon, h}(\sigma, \tau)=\left(\sigma\left(\delta_{\varepsilon}^{i} \times 1\right), \tau \delta_{\varepsilon}^{i}\right), \quad \partial_{j}^{\varepsilon, v}(\sigma, \tau)=\left(\sigma\left(1 \times \delta_{\varepsilon}^{j}\right), \tau\right), \quad \varepsilon \in\{0,1\}, \\
s_{i}^{h}(\sigma, \tau)=\left(\sigma\left(\eta^{i} \times 1\right), \tau \eta^{i}\right), \quad s_{j}^{v}(\sigma, \tau)=\left(\sigma\left(1 \times \eta^{j}\right), \tau\right),
\end{gathered}
$$

(see Example 2.10) form a bicubical set which we denote by $Q(f)$. By Proposition 8.2, the free bicubical abelian group $\mathbb{Z}[Q(f)]$ yields two spectral sequences. The second spectral sequence collapses and the first one is the Serre spectral sequence of $E \stackrel{f}{\longrightarrow} B$. Note that this construction of the Serre spectral sequence is the original one used by Serre in $[\mathbf{2 1}]$.

Now, using Theorem 1.1, we compare the cubical and simplicial singular homologies of a topological space. Let $\Delta^{n}, n \geqslant 0$, denote the standard $n$-simplex. The class $\mathscr{P}_{\Delta}$ of all possible disjoint unions of standard simplices is a projective class (in the sense of Definition 4.1) in the category Top of topological spaces. (Moreover, in fact, it comes from a cotriple $[\mathbf{3},(10.2)]$.) Indeed, for any space $Y$, the map

$$
\bigsqcup_{\substack{\Delta, n \rightarrow Y, n \geqslant 0}} \Delta^{n} \rightarrow Y
$$

where the disjoint union is taken over all possible continuous maps $\Delta^{n} \rightarrow Y, n \geqslant 0$, is a $\mathscr{P}_{\Delta}$-epimorphism. Consider the functor

$$
F: \mathbf{T o p} \rightarrow \mathbf{A b}, \quad F(Y)=H_{0}^{\Delta}(Y, A)=\mathbb{Z}\left[\pi_{0} Y\right] \otimes A,
$$

where $\mathbf{A} \mathbf{b}$ is the category of abelian groups, $H_{*}^{\Delta}(Y, A)$ the simplicial singular homology of $Y$ with coefficients in an abelian group $A$, and $\pi_{0} Y$ the set of path components of $Y$. It follows from $[\mathbf{3},(10.2)]$, and $[\mathbf{2 4},(3.1)]$ that there is a natural isomorphism

$$
\mathbf{L}_{n}^{\Delta} F(Y) \cong H_{n}^{\Delta}(Y, A), \quad n \geqslant 0,
$$

where the simplicial derived functors are taken with respect to the projective class $\mathscr{P}_{\Delta}$ (cf. [19], [20]). We sketch the proof of this natural isomorphism along the lines of $[\mathbf{3}$, (10.2)]. The standard cosimplicial object $\Delta^{\bullet}$ gives rise to an augmented simplicial functor

$$
F_{\bullet}^{\Delta} \rightarrow F, \quad F_{n}^{\Delta}(Y)=\mathbb{Z}\left[\operatorname{Hom}_{\text {Top }}\left(\Delta^{n}, Y\right)\right] \otimes A .
$$

Further, suppose $S \rightarrow Y$ is a $\mathscr{P}_{\Delta}$-projective presimplicial resolution of $Y$. Evaluating $F_{\bullet}^{\Delta}$ on $S$ yields a bipresimplicial abelian group. It is easily seen that both resulting spectral sequences collapse at $E^{2}$ and give the desired isomorphism.

Similarly, one can describe the cubical singular homologies $H_{n}^{\square}(Y, A), n \geqslant 0$, as cubical derived functors of the functor $F(Y)=\mathbb{Z}\left[\pi_{0} Y\right] \otimes A$. To show this we use the 
class $\mathscr{P}_{\square}$ consisting of all possible disjoint unions of standard cubes. The class $\mathscr{P}_{\square}$ is a projective class in Top and there is a natural isomorphism

$$
\mathbf{L}_{n}^{\square} F(Y) \cong H_{n}^{\square}(Y, A), \quad n \geqslant 0,
$$

where the cubical derived functors are taken with respect to $\mathscr{P}_{\square}$. Indeed, the standard cocubical object $I^{\bullet}$ (see Example 2.10) yields an augmented cubical functor

$$
F_{\bullet}^{\square} \rightarrow F, \quad F_{n}^{\square}(Y)=\mathbb{Z}\left[\operatorname{Hom}_{\mathbf{T o p}}\left(I^{n}, Y\right)\right] \otimes A .
$$

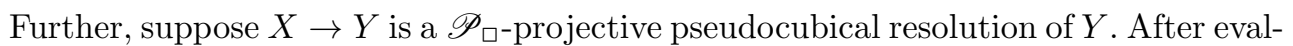
uating $F_{\bullet}^{\square}$ on $X$ we get a bipseudocubical abelian group $F_{\bullet}^{\square}(X)$. By Proposition 8.2, we have two spectral sequences associated to $F_{\bullet}^{\square}(X)$. Both spectral sequences have only one possible non-trivial row at the $E^{2}$-stage and thus they collapse, giving natural isomorphisms

$$
H_{n}^{\square}(Y, A) \cong H_{n}\left(\operatorname{Tot} N N F_{\bullet}^{\square}(X)\right), \quad n \geqslant 0,
$$

and

$$
\mathbf{L}_{n}^{\square} F(Y) \cong H_{n}\left(\operatorname{Tot} N N F_{\bullet}^{\square}(X)\right), \quad n \geqslant 0 .
$$

Combining these two isomorphisms we get the desired isomorphism.

Note that the class $\mathscr{P}=\mathscr{P}_{\Delta} \cup \mathscr{P}_{\square}$ is also a projective class in Top. Obviously, the simplicial derived functors with respect to the class $\mathscr{P}_{\Delta}$ are naturally isomorphic to the simplicial derived functors with respect to $\mathscr{P}$. Similarly, the cubical derived functors with respect to the class $\mathscr{P}_{\square}$ are naturally isomorphic to the cubical derived functors with respect to $\mathscr{P}$. Thus, by 1.1, there is a natural isomorphism

$$
\mathbf{L}_{n}^{\Delta} F(Y) \cong \mathbf{L}_{n}^{\square} F(Y), \quad n \geqslant 0,
$$

for any topological space $Y$, i.e.,

$$
H_{n}^{\Delta}(Y, A) \cong H_{n}^{\square}(Y, A), \quad n \geqslant 0 .
$$

\section{Acknowledgements}

This research was partially supported by the Deutsche Forschungsgemeinschaft Graduiertenkolleg 1150 "Homotopy and Cohomology".

\section{References}

[1] R. Antolini, B. Wiest, The singular cubical set of a topological space, Math. Proc. Camb. Philos. Soc. 126 (1999), no. 1, 149-154.

[2] M. Barr, J. Beck, Acyclic models and triples, Proc. Conf. Categor. Algebra, La Jolla 1965 (1966), 336-343.

[3] M. Barr, J. Beck, Homology and standard constructions, Lecture notes in mathematics 80, Springer, 1969, 245-335.

[4] R. Brown, P. J. Higgins, On the algebra of cubes, J. Pure Appl. Algebra 21 (1981), 233-260.

[5] R. Brown, P. J. Higgins, Cubical Abelian groups with connections are equivalent to chain complexes, Homology Homotopy Appl. 5 (2003), no. 1, 49-52. 
[6] A. Dold, D. Puppe, Homologie nicht-additiver Funktoren. Anwendungen, Ann. Inst. Fourier 11 (1961), 201-312.

[7] S. Eilenberg, J. C. Moore, Foundations of relative homological algebra, Mem. Amer. Math. Soc. 55 (1965).

[8] P. J. Freyd, Abelian categories, an introduction to the theory of functors, Harper \& Row, New York, Evanston, London, 1964.

[9] P. Goerss, J. F. Jardine, Simplicial homotopy theory, Progress in Mathematics 174, Birkhäuser, Basel, 1999.

[10] M. Golasinski, Homotopies of small categories, Fundam. Math. 114 (1981), no. 3, 209-217.

[11] S. B. Isaacson, Cubical homotopy theory and monoidal model categories, $\mathrm{PhD}$ thesis, Harvard University, 2009, available online at http://www.ma.utexas.edu/users/isaacson/PDFs/diss.pdf.

[12] T. Kadeishvili, S. Saneblidze, A cubical model for a fibration, J. Pure Appl. Algebra 196 (2005), no. 2-3, 203-228.

[13] D. Kan, Abstract homotopy I, Proc. Natl. Acad. Sci. USA 41 (1955), 10921096.

[14] D. Kan, Abstract homotopy II, Proc. Natl. Acad. Sci. USA 42 (1956), 255-258.

[15] M. Karoubi, K-theory, an introduction, Springer-Verlag, Berlin, Heidelberg, New York, 1978.

[16] M. Levine, Smooth motives, Fields Institut Communications 56, Motives and Algebraic Cycles: A Celebration in Honour of Spencer J. Bloch, (2009), 175232.

[17] W. S. Massey, Singular homology theory, Springer-Verlag, Berlin, New York, 1980.

[18] B. Mitchell, Rings with several objects, Advances in Math. 8 (1972), 1-161.

[19] G. S. Rinehart, Satellites and cohomology, J. Algebra 12 (1969), 295-329.

[20] G. S. Rinehart, Singular homology as a derived functor, Trans. Amer. Math. Soc. 174 (1972), 243-256.

[21] J. P. Serre, Homologie singuliere des espaces fibres, Annals of Math. 54 (Nov., 1951), no. 3, 425-505.

[22] A. Świątek, Kategorie obiektów kostkowych, $\mathrm{PhD}$ Thesis, preprint nr 27, Toruń, 1975.

[23] A. Świątek, Category of cubical objects and category of simplicial objects, Comment. Math. Prace Mat. 22 (1980/81), no. 2, 307-316.

[24] M. Tierney, W. Vogel, Simplicial resolutions and derived functors, Math. Zeit. 111 (1969), 1-14.

[25] A. P. Tonks, Cubical groups which are Kan, J. Pure Appl. Algebra 81 (1992), no. $1,83-87$.

[26] C. A. Weibel, An introduction to homological algebra, Cambridge Studies in Advanced Mathematics. 38., Cambridge Univ. Press, Cambridge, 1994. 
Irakli Patchkoria irpatchk@math.uni-bonn.de

Mathematisches Institut, Universität Bonn, Endenicher Allee 60, 53115 Bonn, Germany 